\title{
Contributions to Techniques for Learning Non-reactive Behaviour from Observation
}

by

\section{Caleb Chan}

\author{
A Thesis submitted to \\ the Faculty of Graduate Studies and Research \\ in partial fulfilment of \\ the requirements for the degree of \\ Master of Applied Science \\ Ottawa-Carleton Institute for \\ Electrical and Computer Engineering
}

Department of Systems and Computer Engineering

Carleton University

Ottawa, Ontario, Canada

December 2015 
Copyright (c)

2015 - Caleb Chan 


\section{Abstract}

Learning from observation allows an expert to train a software agent or robot without explicitly programming the behaviour. Behaviour learned can be broken down into categories: reactive and non-reactive. Actions in reactive behaviour are based on the current environment state whereas actions in non-reactive uses both current state, and any past action or states. We will analyze and compare using a common benchmark two recent and partially studied approaches to learning non-reactive behaviour from observation: Dynamic Bayesian Networks (DBN) and Temporal Backtracking (TB). The goal is to characterize situations where one approach should be preferred over the other.

We will attempt to provide a more general case-based reasoning framework for non-reactive behaviour learning. Using the framework and the benchmark, we will analyze and compare three different metrics for comparing cases: run similarity, edit distance and Jaccard distance. This will allow characterization of situations where one metric should outperform the other. 


\section{Table of Contents}

$\begin{array}{ll}\text { Abstract } & \text { iii }\end{array}$

Table of Contents $\quad$ iv

List of Tables vii

List of Figures $\quad$ xi

1 Introduction 1

1.1 Motivation . . . . . . . . . . . . . . 5

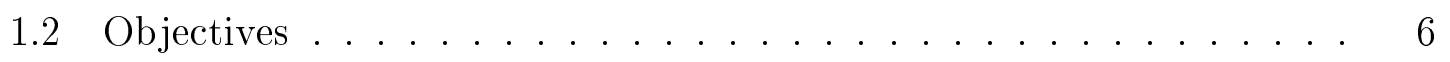

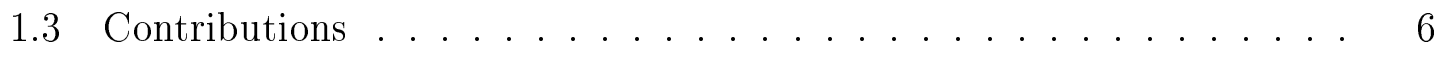

1.4 Organization $\ldots \ldots \ldots \ldots \ldots \ldots \ldots \ldots \ldots \ldots \ldots \ldots \ldots \ldots$

2 Background $\quad 9$

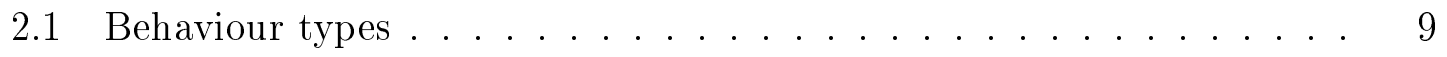

2.2 Bayesian Network . . . . . . . . . . . . . . . . 11

2.3 Dynamic Bayesian Network . . . . . . . . . . . . . . . 15

2.4 Expectation Maximization . . . . . . . . . . . . 18 
2.5 Case-Based Reasoning . . . . . . . . . . . . . 20

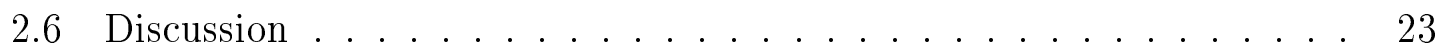

3 State of the Art 25

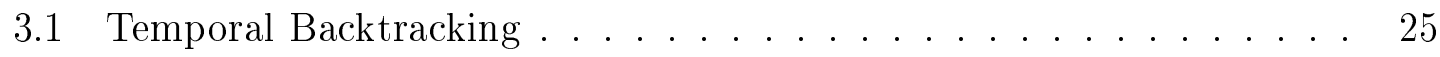

3.2 Dynamic Bayesian Network _. . . . . . . . . . . . . . . 30

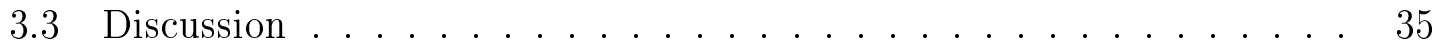

4 Analysis of Existing Approaches $\quad 37$

4.1 Learning from Observation using a Dynamic Bayesian Network . . . . 38

4.2 TB to Infer Internal State . . . . . . . . . . . . . . . 45

4.2.1 TB Using Non-deterministic Candidate Run Selection . . . . . 48

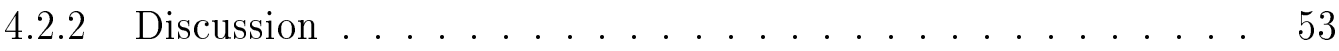

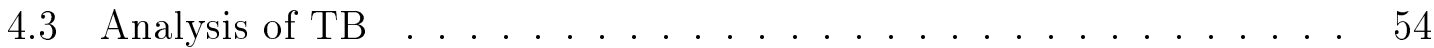

4.3.1 Consensus not reached at the end of the problem run . . . . 55

4.3.2 Time Slice versus Run Similarity . . . . . . . . . . 56

4.3.3 Length of Candidate run (Cut-off point) . . . . . . . . 60

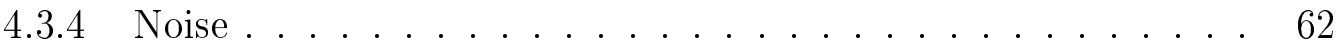

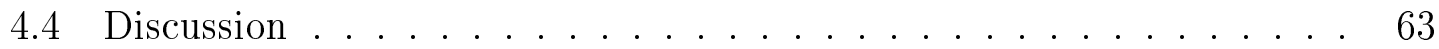

5 Methodology and Results of Generalizing the Case-based Reasoning Approach to Case Similarity $\quad 65$

5.1 Defining the Framework . . . . . . . . . . . . 65

5.2 Run Similarity . . . . . . . . . . . . . . . . 68

5.2.1 Comparison With TB ............... 70 
5.2 .2 Constant Decay . . . . . . . . . . . . 75

5.2 .3 Exponential Decay .................. 80

5.2 .4 Gaussian Decay . . . . . . . . . . . . . . 84

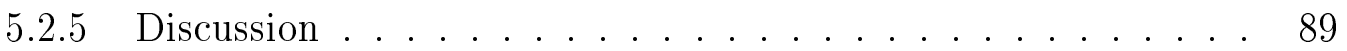

5.3 Edit distance calculation . . . . . . . . . . . . . 89

5.4 Jaccard Distance Calculation _. . . . . . . . . . . 99

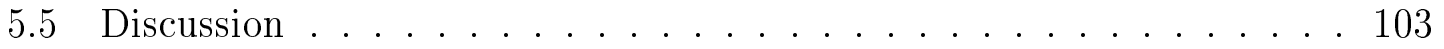

6 Conclusion $\quad 106$

6.1 Summary of Results / Contributions . . . . . . . . . . . 106

6.2 Future Work . . . . . . . . . . . . . 107

$\begin{array}{ll}\text { List of References } & 109\end{array}$ 


\section{List of Tables}

Table 1 Original results [1] showing the accuracy of Bayesian network $(\mathrm{BN})$, neural network $(\mathrm{NN})$, sliding window version of $\mathrm{BN}$ and $\mathrm{NN}$ where $k=2$ (BNk2 and NNk2), input-output hidden markov model (IOHMM), and dynamic Bayesian network (DBN) . . . . . . 34

Table 2 A comparison of the original accuracies shown in the top table [1] versus the retested results in the bottom two tables. The tables show the values of Bayesian network (BN), neural network (NN), sliding window version of $\mathrm{BN}$ and $\mathrm{NN}$ where $k=2$ (BNk2 and NNk2), input-output hidden markov model (IOHMM), and dynamic Bayesian network (DBN). The experiment was done using 7 -fold cross validation, where each fold is 1000 steps long; this was repeated for six different datasets. Bold values are either above or below their original values if we factor in the margin of error $\ldots \ldots \ldots . \ldots 39$ 
Table 3 Average F1 measure of the retested results. The tables show the values of Bayesian network (BN), neural network (NN), sliding window version of $\mathrm{BN}$ and $\mathrm{NN}$ where $k=2(\mathrm{BNk} 2$ and $\mathrm{NNk} 2)$, inputoutput hidden markov model (IOHMM), and dynamic Bayesian network $(\mathrm{DBN}) . \ldots \ldots \ldots \ldots \ldots$

Table 4 The average time in seconds needed to train the graphical network (Bayesian network $(\mathrm{BN})$, neural network (NN), sliding window version of BN and NN where $k=2$ (BNk2 and NNk2), inputoutput hidden markov model (IOHMM), and dynamic Bayesian network $(\mathrm{DBN})) \ldots \ldots \ldots \ldots \ldots \ldots$

Table 5 The average time in milliseconds that the graphical network (Bayesian network $(\mathrm{BN})$, neural network $(\mathrm{NN})$, sliding window version of BN and NN where $k=2(\mathrm{BNk} 2$ and $\mathrm{NNk} 2)$, input-output hidden markov model (IOHMM), and dynamic Bayesian network (DBN)) take to predict the action once they have respectively received the environment state ......................... 44

Table 6 Accuracy (Top) and F1-Measure (Bottom) of the temporal backtracking algorithm for $k$ values $4,10,20$, and $100 \ldots \ldots$

Table 7 Average run time (in milliseconds) of the Temporal Backtracking

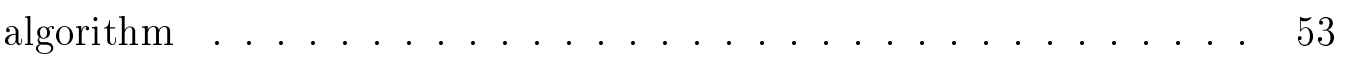

Table 8 The similarity matrix for inputs ........... 55

Table 9 The similarity matrix between inputs . . . . . . . . 57 
Table 10 Breakdown over a run of similarity calculations for temporal backtracking algorithm and run similarity with constant decay weight function where $C=0.1 \ldots \ldots . \ldots 71$

Table 11 Breakdown over a run of similarity calculations for temporal backtracking algorithm and run similarity with constant decay weight function where $C=0.1 \ldots \ldots \ldots 72$

Table 12 Breakdown over a run of similarity calculations for temporal backtracking algorithm and run similarity with constant decay weight function where $C=0.1 \ldots \ldots \ldots \ldots 74$

Table 13 Run Similarity Accuracy of Time Dependent $k=10$ with con-

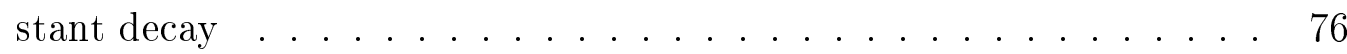

Table 14 Run Similarity F1-measure of Time Dependent k = 10 with constant decay . . . . . . . . . . . . . . . 77

Table 15 Average run time (in milliseconds) of Temporal Backtracking with run similarity using a constant weight function . . . . . . . 79

Table 16 Run Similarity Accuracy of Exponential Decay $k=10$ compared against the temporal backtracking algorithm (TB) . . . . . . . . 81

Table 17 Run Similarity F1-measure of Exponential Dependent k = 10 with constant decay . . . . . . . . . . . . . 82

Table 18 Average run time (in milliseconds) of Temporal Backtracking with run similarity using an exponential weight function . . . . . 83

Table 19 Run Similarity Accuracy of Gaussian Decay $k=10$ compared against the temporal backtracking algorithm (TB) . . . . . . 85 
Table 20 Run Similarity F1-measure of Gaussian Dependent k = 10 with constant decay ......................... 86

Table 21 Average run time (in milliseconds) of Temporal Backtracking with run similarity using a Gaussian weight function . . . . . . 88

Table 22 Run comparison of the first example. The bold values are the final minimum amount of edits for each candidate run . . . . . . . . . 93

Table 23 Comparison of temporal backtracking algorithm to temporal backtracking with edit distance similarity . . . . . . . . . . 93

Table 24 Run comparison of the second example. The bold values are the final minimum amount of edits for each candidate run . . . . . . 94

Table 25 Comparison of temporal backtracking algorithm to edit distance 95

Table 26 Result for edit distance calculation compared against the temporal backtracking algorithm (TB). Accuracy and F1-measure of the TB algorithm are at $k=10 \ldots \ldots . \ldots . \ldots 96$

Table 27 Run time milliseconds of the Temporal Backtracking algorithm with edit distance .................... 96

Table 28 Edit distance F1 score for each action . . . . . . . . . . 99

Table 29 Result for Jaccard distance calculation compared against the temporal backtracking algorithm (TB). Accuracy and F1-measure of the TB algorithm are at $k=10 \ldots \ldots$. . . . . . . . 101

Table 30 Run time milliseconds of the Temporal Backtracking algorithm with Jaccard distance . . . . . . . . . . . . . . . . 101

Table 31 Comparison of F1 measure of each of the actions for Jaccard and Edit distance metrics f . . . . . . . . . . . . 102 


\section{List of Figures}

Figure 1 The interaction between the agent and its environment . . . 3

Figure 2 Example of Bayesian network . . . . . . . . . . 13

Figure 3 Example of Bayesian network using past information . . . . . 14

Figure 4 The breakdown of non-reactive example into independent training examples........................... 14

Figure 5 A simple Dynamic Bayesian Network example showing the state dependencies between states in a time slice and between slices . 16

Figure $6 \quad$ A two slice Dynamic Bayesian Network where $S_{t}$ is the hidden state at time $t$, and $Y_{t}$ is the observed output at time $t . \ldots . . .19$

Figure 7 A visual representation of the four-step cycle of case-based

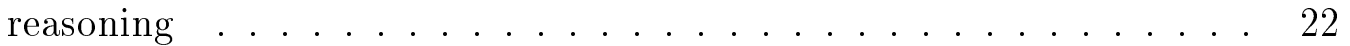

Figure 8 Shown on the left is the configuration of the eight sensors, four dirt and four range, used with the vacuum robot. Shown on the right is a tuple representing the binary information from each of the sensors. $N_{D}$ represents the north dirt sensor and $N_{R}$ represents the north range

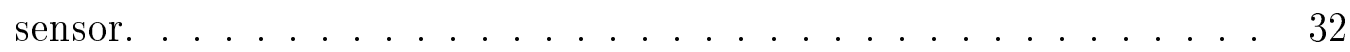


Figure 9 The agent (A), dirt (D) and an obstruction (opaque squares). Shown on the right is the corresponding sensory tuple, demonstrating the sensory data from each of the eight sensors. . . . . . . . . . 32

Figure 10 A class diagram showing the relationships between cases and

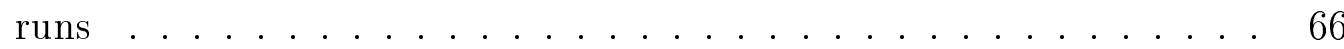

Figure 11 A class diagram showing the relationship between cases and similarity strategies used for case comparison . . . . . . . . . . 67 


\section{Chapter 1}

\section{Introduction}

There are many situations where an intelligent agent's or a robot's behaviour cannot be programmed in advance due to the environment not being known. Some examples are a search-and-rescue robot being deployed in an unknown environment, or a robot assisting the elderly not having the correct treatment procedures. Different environments or procedures require different behaviours, so programming the robot to handle all situations may not be possible. One solution to this problem would be to reprogram the robot when the environment or the procedure has changed. A person who may have the expertise to deal with the environment or know the correct procedure may not have the technical knowledge to reprogram the robot. Another solution would be to demonstrate the correct procedure or how to behave in the environment and have the robot learn. This type of learning is called Learning from Observation (LFO), and allows experts with no technical programming skills to program robots by demonstrating the correct behaviour.

LFO uses the interaction between an agent and its environment and attempts to learn the agent's behaviour. The purpose of LFO is to learn the behaviour of 
an agent by observing the agent's solutions to different situations with the goal of producing a similar solution under a similar situation. LFO can be done with the end goal of either imitating the agent or predicting its behaviour [2]. In the case of the search and rescue mission, the robot can learn the actions required to traverse over obstacles and imitate those actions when it encounters the same obstacle. In the assistance to the elderly situation, the robot can learn the habits of the elderly person and predict their actions to help accommodate them better.

LFO is broken down into two steps: policy derivation and policy execution [3]. In the policy derivation step, the learner observes an agent interacting with the environment and the learner will attempt to derive the agent's estimated behaviour. In the second step, policy execution, the learner uses the derived behaviour to interact with the same environment as the agent. The actions taken in this step are highly dependent on the behaviour in the derivation step and correct behaviour derivation is crucial.

The agents being learned from are humans or autonomous computer systems that can perform actions in a given environment to achieve a goal or series of goals [4]. The environment in which the agent resides can be in any state from a finite number of discrete instantaneous states.

$$
E=\left\{e, e^{\prime}, e^{\prime \prime}, \ldots\right\}
$$

An agent can perform an action from a set number of finite actions to change the state of the environment.

$$
A c=\left\{a, a^{\prime}, a^{\prime \prime}, \ldots\right\}
$$




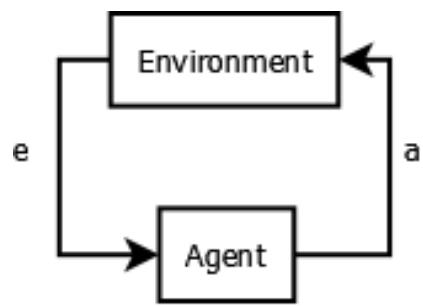

Figure 1: The interaction between the agent and its environment

An agent interacting with the environment proceeds as follows. The environment starts out in an initial state. Based on that initial state, the agent selects an action from its set of actions to further its goal. After the environment has responded to the action, the agent can now select another action based on the current state of the environment. As with the first action, the environment can change its state based on the action by the agent. This back and forth between the environment and agent goes on and on in a feedback loop shown in Figure 1.

The back and forth between the environment and the agent can be represented by a run, $r$.

$$
r: e \stackrel{a}{\rightarrow} e^{\prime} \stackrel{a^{\prime}}{\rightarrow} e^{\prime \prime} \stackrel{a^{\prime \prime}}{\rightarrow} e^{\prime \prime \prime}
$$

We can use runs to derive state-based behaviour based on the mapping between the environment and actions. Let us consider a trivial example of a floor-cleaning agent that turns right when it hits a wall, but otherwise moves forward. Let us look at a sample run of the agent:

$$
r: \text { Space } \stackrel{\text { forward }}{\rightarrow} \text { Wall } \stackrel{\text { right }}{\rightarrow} \text { Wall } \stackrel{\text { right }}{\rightarrow} \text { Space } \stackrel{\text { forward }}{\rightarrow}
$$

From the run, we can see that the agent moves forward only if there is space and will turn right only if there is a wall. From this run, we can see that the agent's behaviour is only dependent on the last state of the environment. 
Wooldridge [4] defines a reactive agent as an agent that will respond to the current state of the environment with no reference to the past. A reactive agent, $A g$, can be expressed in the following function for the agent:

$$
A g: E \rightarrow A c
$$

where $E$ is the set of states of the environment and $A c$ is the set of resulting actions of the agent, given the state information. The agent's actions are governed by the state of the environment, and each state of the environment returns the same action. In the previous example the actions of forward and right were only based on the environment states Space and Wall respectively. As a result, from the run we can say that the agent that produced the run is a reactive agent. A major portion of work on LFO has focused on learning such reactive behaviour [3] [5] [6].

Now let us use the same agent, but change its turn behaviour to alternate between turning left and turning right. If we look at a sample of a run from the agent, it might look like this:

$$
r: \text { Space } \stackrel{\text { forward }}{\rightarrow} \text { Wall } \stackrel{\text { right }}{\rightarrow} \text { Wall } \stackrel{\text { left }}{\rightarrow} \text { Space } \stackrel{\text { forward }}{\rightarrow}
$$

As with the first example, we see that the agent will only move forward if there is space. If we look at the behaviour of the agent when it hits a wall, we see it performs one of two possible actions, turning left or right. Having two possible actions for one environment state does not satisfy the definition of a reactive agent. We can denote agents that do not satisfy the definition of a reactive agent as non-reactive or memory-based. Because there are two actions for one environment state, the agent is using other information besides the current environment state to decide which of 
the two actions should be taken. This tells us that the agent is using some internal state (not to be confused with the environment state) to select the next action. The internal state is remembering specific information from the past. In the example above, the agent is remembering the last action it took.

\subsection{Motivation}

The purpose of LFO is to learn the behaviour of an agent by observing the it, with the goal of producing a similar output when given a similar input. Learning of a reactive agent involves finding which output corresponds to which input. Because the actions of a reactive agent are based on the input, each input only has one action associated with it.

Learning the behaviour of a non-reactive agent is more complicated due to the need for an internal memory, I. Actions are dependent on the state of the environment and the internal state. Taking the internal state into account, mapping environment states to actions becomes $\{E \times I\}: A$. This mapping holds true only if we have access to the internal state. If we do not have access to the internal state, we have to infer the value of $I$.

This leads to the difficultly of modelling the internal state. We can model the internal state by representing the internal state as a variable. This variable can be used to represent a summary of past information. We can also model the internal state by using all or a subset of past inputs and output. These different models leads us to wonder which model is the better model and why. 


\subsection{Objectives}

In this thesis we will look into two principal existing approaches to learning nonreactive behaviour from observation. One approach relies on graphical models such as Dynamic Bayesian Networks (DBN) [1] while the other uses a technique called Temporal Backtracking (TB) [7]. Both approaches are very recent and have not been studied or validated throughly yet. Our goal then is to analyze and compare these approaches using a common benchmark, and attempt to characterize situations where one approach should be preferred over the other. Furthermore, we will attempt to provide a more general case-based reasoning framework for non-reactive behaviour learning.

\subsection{Contributions}

The key contributions of the work are:

- An analysis of previous research using a stochastic solution for LFO [1]. From our attempts at reproducing their results, problems with the quality of the results were found, including missing variances and the use of accuracy over F-measure. By reproducing the results, we were able to provide the missing metrics, resulting in a baseline to measure other solutions for learning from observation (Section 4.1).

- A comparative analysis of a case-based solution (temporal backtracking) [7] against the stochastic solution using the benchmark provided by Ontañón et al. [1]. This resulted in the conclusion that for highly complex behaviour, the 
stochastic solution is better (Section 4.2).

- An in-depth analysis of the temporal backtracking algorithm. From the analysis, we identified situations that resulted in behaviour not defined from the original definition of the temporal backtracking algorithm. These situations included the algorithm not being able to find a solution, premature elimination of solutions, and an inability to handle variable candidate run lengths. Additionally, through the analysis, it was concluded that the temporal backtracking algorithm was only one possible case-based reasoning solution for LFO that could be generalized (Section 4.3).

- A framework that allows for a generalized solution that allows for a variety of similarity metrics to be applied on runs or subsections of runs(Section 5.1).

- Analysis of three different similarity metrics, run similarity (Section 5.2), edit distance (Section 5.3) and Jaccard distance (Section 5.4) as an alternative to the temporal backtracking algorithm. From the analysis, it was shown that these similarity metrics have little to no improvement over the temporal backtracking algorithm.

\subsection{Organization}

Chapter 2 will provide background information about the reactive and non-reactive behaviour mentioned earlier in the introduction. In Chapter 3, we will be looking at two solutions, stochastic and case-based for LFO.

The major contribution of this thesis will be presented in Chapters 4 and 5 . 
Chapter 4 will analyze the two solutions for LFO. In this chapter, we will break down some of the problems with the case-based solution and propose some solutions for theses problems.

In Chapter 5, a new framework will be introduced and defined as a generalized solution to the temporal backtracking analyzed in Chapter 4. Using this new general framework, the chapter will also introduce three approaches to attempt to improve on the temporal backtracking solution.

A summary of the work performed and concluding remarks will be discussed in Chapter 6 . 


\section{Chapter 2}

\section{Background}

\subsection{Behaviour types}

As stated in the Introduction section, LFO involves learning the behaviour of an agent by observing an agent interact with an environment. The behaviours that can be learned can be classified into two families: reactive and non-reactive.

Reactive Behaviour An agent that has reactive behaviour responds to the current state of the environment with no reference to the past. An example of a reactive behaviour is an agent that will turn right when it hits a wall and forward if it does not hit a wall. We have previously discussed the notion of a reactive agent, and it is expressed in Equation 1.

$$
A g: E \rightarrow A c
$$

Because the agent does not refer to the past when deciding on the next action, each environment state leads to only one action by the agent. This results 
in each environment state having a 1:1 mapping with an action. Learning a reactive behaviour involves learning the mapping of the environment state to the action.

An example of a reactive agent would be an vacuum robot that always turns right when it hits a wall. This robot is a reactive agent because the action it takes is dependent on the current state of the environment and does not need any past information.

Non-Reactive/Memory-Based Behaviour An agent with this type of behaviour responds to the current state of the environment and to its internal memory, $I$, when deciding on the next action. The internal memory stores information on past actions or past environment states, and is used by the agent to perform an action in the future. In theory, the agent's memory is limitless and can store an infinite amount of information from the the past. In practice, the memory is limited and only a limited amount of information is stored. The internal memory is constantly being updated when it receives new information from the environment, and actions by the agent are dependent both on the current environment state and the internal memory. Equation 1 describes an agent in which the action is dependent only on the last environment state and it is not adequate for describing non-reactive behaviour. To account for the actions of an agent being dependent on the internal memory, we must declare an action function:

$$
\text { action }: I \rightarrow A c
$$

where $I$ is the internal state or internal memory of the agent. The internal 
memory is updated in the following fashion:

$$
\text { next : } E \times I \rightarrow I
$$

The behaviour of a non-reactive agent is as follows. The agent starts out with an initial state, $I_{0}$. The agent receives new information from the environment and updates the internal state using the $\operatorname{next}(E)$ function. The new internal state is used to decide what the next action should be. Because actions are dependent to the environment state and the internal state, each state of the environment can result in different actions based on the state of $I$.

Learning the behaviour of a non-reactive agent is more complicated due to the inability to observe the internal state, $I$. There has been research done on how to best infer this internal state. This can be done through Case-BasedReasoning (CBR) [2] [8] or through a graphical solutions [9] to name a few. The two solutions reviewed in this thesis are the Dynamic Bayesian Network [1] and the Temporal Backtracking method [7].

\subsection{Bayesian Network}

In the previous section, we discussed the different behaviour types that can be learned from observation. For reactive behaviour, learning the behaviour involves mapping each environment state to an action in a 1:1 mapping. For non-reactive behaviour, the internal state of the agent needs to be inferred. Ontañón et al. [1] suggested a model for a Bayesian network that would allow it to learn reactive behaviour. The 
network can be further extended to allow for a specific type of non-reactive behaviour. In this section, we will introduce Bayesian networks and look at the model used by Ontañón et al.

A Bayesian network is a graphical model that represents a set of random variables and the dependencies among those random variables [10]. Bayesian networks are described using a directed acyclic graph. Each node in the graph represents a variable and variables can be either continuous or discrete. Edges of the graph connect two pairs of nodes together, and if an edge connects node $\mathrm{X}$ to node $\mathrm{Y}$, it is said that node $\mathrm{X}$ is the parent of node $\mathrm{Y}$. Each node has a distribution of $P\left(X_{i} \mid \operatorname{Parent}\left(X_{i}\right)\right)$, which represents the effect of the parents on the current node.

Consider a run of a reactive vacuum robot that will turn left when it hits a wall.

$$
r: \text { NoWall } \stackrel{\text { Forward }}{\rightarrow} \text { NoWall } \stackrel{\text { Forward }}{\rightarrow} \text { Wall } \stackrel{\text { Left }}{\rightarrow} \text { Wall } \stackrel{\text { Left }}{\rightarrow}
$$

Ontañón et al. suggested a model for Bayesian networks that would allow it to learn reactive behaviour. This model is shown in Figure 2 and model can be represented by the distribution $P\left(X_{i}, Y_{i}\right)=P\left(X_{i}\right) P\left(Y_{i} \mid X_{i}\right)$ where $X_{i}$ is the environment state at time $i$ and $Y_{i}$ is the action of the agent at time $i$.

What is interesting about this model is that the distribution $P\left(Y_{i} \mid X_{i}\right)$ can be used to describe the relationship between actions and environment states. As we can see from Figure 2, in the absence of noise the probability of $P\left(Y_{i} \mid X_{i}\right)$ shows us a 1:1 mapping of an environment state to the appropriate action by having one value on each row have the probability of 1 and all other probabilities on the row be 0 .

This model can be further extended to include specific non-reactive behaviours. Consider a vacuum robot that will alternate turn direction when it hits a wall and 

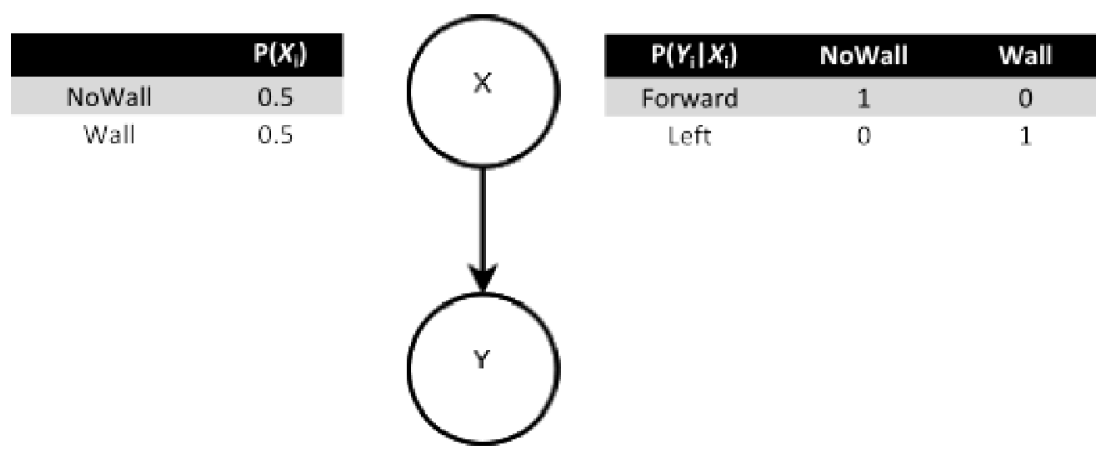

Figure 2: Example of Bayesian network

will turn in the same direction of the robot does not hit a wall. A sample trace would look like the following:

$$
r: \text { NoWall } \stackrel{\text { Left }}{\rightarrow} \text { NoWall } \stackrel{\text { Left }}{\rightarrow} \text { Wall } \stackrel{\text { Right }}{\rightarrow} \text { NoWall } \stackrel{\text { Right }}{\rightarrow} \text { Wall } \stackrel{\text { Left }}{\rightarrow} \text { Wall } \stackrel{\text { Right }}{\rightarrow}
$$

From the trace, we can see that both the NoWall state and the Wall state have two actions associate with them. As a result, the behaviour is non-reactive. Actions by the robot are dependent on the current environment state and the past action. This means that the robot must remember the previous action it took. Since we know that the robot will always use the previous action to and the current environments state to make its action, we can expand the model shown in Figure 2 to include the previous action. This results in the model shown in Figure 3. This model is know as the sliding window approach [11]. The sliding window approach introduces a window classifier around the current input, $X_{i}$, and assumes that all past information used by the agent or robot exists in this window. The size of the window is the past number of $k$ observations. In Figure 3, the size of the window is $k=1$ and uses $Y_{i-1}$ 
in addition to $X_{i}$ to calculate $Y_{i}$. A window with $k=2$ would include the past $Y_{i-1}$ and $X_{i-1}$.

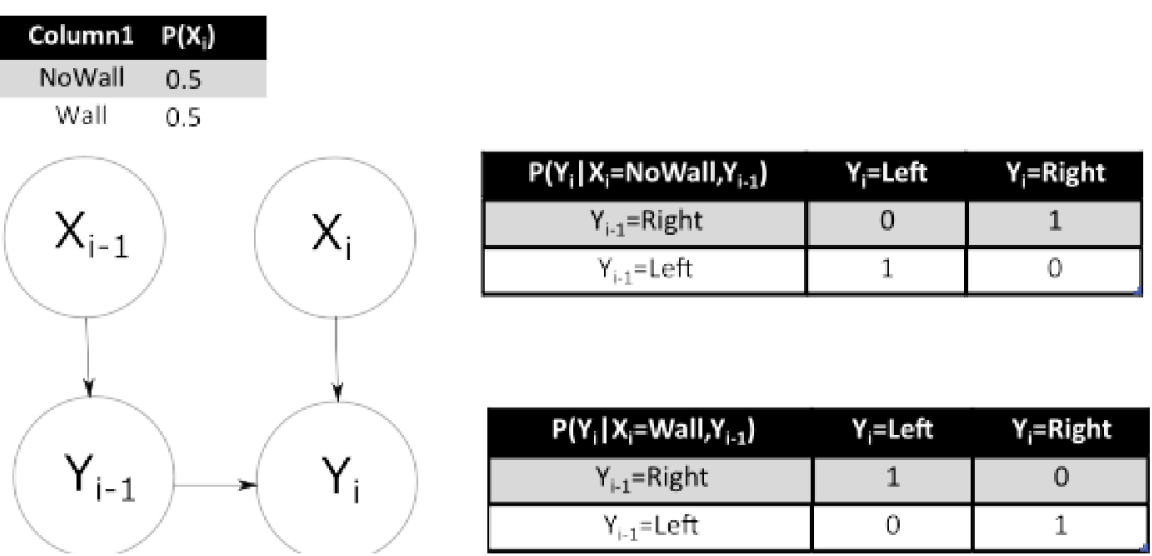

Figure 3: Example of Bayesian network using past information

Using the sliding window approach allows us to reorganize the sequence into independent training examples. This allows us to apply a classical supervised learning technique such as a Bayesian network. The non-reactive example could be broken down into independent training examples shown in Figure 4 . The benefit of using the sliding window approach is that we do not need to represent the internal state of the robot / agent with another variable, but can just use the past $k$ observations.

\begin{tabular}{|l|l|}
\hline Feature & \multicolumn{1}{l|}{ Action } \\
\hline$Y_{i-1} X_{i+1}$ & \multicolumn{1}{c|}{$Y_{i}$} \\
\hline$Y_{i} X_{i+1}$ & $Y_{i+1}$ \\
\hline$Y_{i+1} X_{i+2}$ & \multicolumn{1}{c|}{$Y_{i+2}$} \\
\hline$Y_{i+2} X_{i+3}$ & $Y_{i+3}$ \\
\hline
\end{tabular}

\begin{tabular}{|l|l|}
\hline \multicolumn{1}{|l|}{ Feature } & \multicolumn{1}{c|}{ Action } \\
\hline , NoWall & \multicolumn{1}{c|}{ Left } \\
\hline Left, NoWall & Left \\
\hline Left, Wall & \multicolumn{1}{|c|}{ Right } \\
\hline Right, NoWall & Right \\
\hline Right, Wall & Left \\
\hline Left, Wall & Right \\
\hline
\end{tabular}

Figure 4: The breakdown of non-reactive example into independent training examples 
In this section we showed how a Bayesian network can be modelled to imitate a reactive agent's behaviour. In addition, by using the sliding window approach, a run sequence can be converted into a list of independent training example and can be solved using a classic supervised learning technique such as a Bayesian network. In the next section, we will introduce another model for learning non-reactive behaviour.

\subsection{Dynamic Bayesian Network}

A Dynamic Bayesian Network (DBN) is a graphical representation of a random process dependent on time [12], composed of a series of time slices that represent instances in time. Each time slice contains a set of random variables at a given instance in time. Time slices can have different random variables between slices, but for the purposes of simplification, we will consider each time slice to be identical. Variables in a time slice can be either hidden or observable. An observable variable is where the values are known; for example, the output of the DBN or the input to the DBN.

Consider the problem of learning the behaviour of a non-reactive robot. Because the robot is non-reactive, it uses an internal memory to make its decisions and we assume that the internal memory values are hidden. We can model this internal state as one random variable, $S$. The internal memory can also be modelled as a number of random hidden or observed variables, but for the purposes of this thesis, we will model the internal state as one random variable. We assume that $S$ can take $m$ number of discrete states. The value of $S$ is dependent on the input from the environment, the previous value of $S$ and the last action. We can model this using 


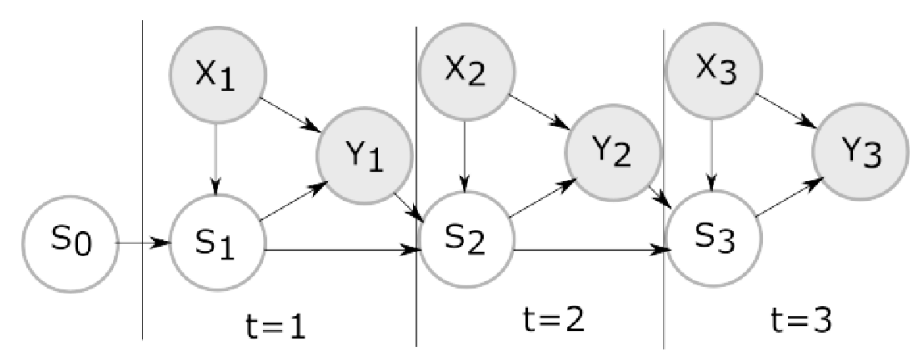

Figure 5: A simple Dynamic Bayesian Network example showing the state dependencies between states in a time slice and between slices

a simple DBN structure, as shown in Figure 5. In the figure at instant $i, X_{i}$ is the input, $Y_{i}$ is the output, $S_{i}$ is the model of the internal memory. In addition, the shaded nodes are observable nodes and the non-shaded nodes are the hidden nodes.

A Hidden Markov model (HMM) is another graphical model and shares similar properties to DBN. A HMM can be represented by a DBN, but a DBN cannot be represented by a HMM. The main difference between a HMM and DBN is that an HMM does not have an $X_{i}$ node.

Three important pieces of information are needed before any type of inference can be made on the DBN: the prior distribution of the state variable, the transition model and the observation model [10]. Using the example of Figure 5, the prior distribution of the state variable is the prior distribution of the internal memory and is given by $P\left(S_{0}\right)$. The transition model would be $P\left(S_{t+1} \mid S_{t}, X_{t+1}, Y_{t}\right)$ and the observation model would be $P\left(Y_{t} \mid S_{t}, X_{t}\right)$. Because all time slices are identical, the transition model and the observation model are the same for all slices. The prior probability is used for the first slice since there exists no slice before the first slice.

If the prior probability, the transition model and the observation model values are known, inference can be done on the DBN. Inference in a DBN can be done in 
one of 4 ways; filtering, prediction, smoothing, and most likely explanation.

Filtering: Filtering is used to calculate the belief state or the posterior probability at time $t$ using the evidence from time 1:t. This means calculating $P\left(S_{t} \mid Y_{1: t}, X_{1: t}\right)$ or probability of the internal memory being in one of the $m$ states when given the evidence (inputs and action from time 1 to $t$ ). To calculate the belief state for time $t$, the previous belief state at time $t-1$ needs to be calculated first. As a result to calculate $P\left(S_{t} \mid Y_{t-1}, X_{t}, S_{t-1}\right), P\left(S_{t-1} \mid Y_{1: t-2}, X_{1: t-1}, S_{t-2}\right)$ must be calculated first. This calculation is done recursively until $t=0$ is reached. At this point, the value of each belief state from 1:t is propagated forward. This is also known as forward propagation.

Prediction: Prediction is used to calculate the distribution at a future state using the evidence from time 1:t. This is calculated using $P\left(S_{t+k} \mid Y_{1: t}, X_{1: t}\right)$ and with $k$ being some state in the future.

Smoothing: Smoothing is used to calculate the distribution over a past state, given the evidence from time 1:t. This is calculated with $P\left(S_{t-k} \mid Y_{1: t}, X_{1: t}\right)$ and $0 \leq$ $k<t$.

Most Likely Explanation: Most likely explanation is used to calculate the most likely sequence through the DBN based on the observations made. This is calculated with $P\left(S_{1: t} \mid Y_{1: t}, X_{1: t}\right)$.

For inference in DBN, the state variable probabilities must be known. When probabilities are not known, they need to be estimated using the Expectation Maximization (EM) algorithm, which is presented in the next section. 


\subsection{Expectation Maximization}

The expectation maximization (EM) algorithm is used to train the unknown parameters of a DBN. The EM algorithm uses the training data and estimates the values of the parameters. The algorithm is broken down into two major steps: the expectation step and the maximization step. The expectation step calculates the log likelihood of $P\left(S_{T} \mid W_{1: T}\right)$ given all the observations, the initial prior probability, transition model and observation model of the system. If any of the initial prior probability, transition model and observation model of the system is unknown, random values are assigned to each. The maximization step recalculates the initial prior probability, transition model and observation model.

The expectation and maximization steps can be repeated until the prior probability, transition model and observation model converge to values that will produce the training data. The stopping criteria for the EM algorithm can be a set number of repetitions and / or repeating until the change in log likelihood of the prior, transition and observation models between iterations is below a given threshold. 


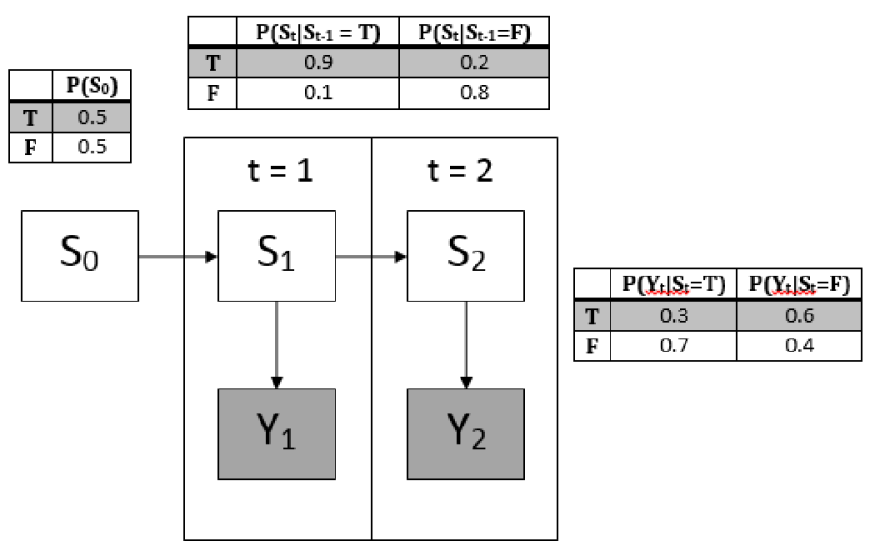

Figure 6: A two slice Dynamic Bayesian Network where $S_{t}$ is the hidden state at time $t$, and $Y_{t}$ is the observed output at time $t$.

For example, we wish to train the DBN shown in Figure 6 using the output $Y_{1}=F$ and $Y_{2}=T$. In the example, the initial prior probability, transition model $\left(P\left(S_{t} \mid S_{t-1}\right)\right)$ and observation model $\left(P\left(Y_{t} \mid S_{t}\right)\right)$ are given. The initial values for the prior probability, transition model and observation model can be randomly generated because the values will be adjusted to fit the training data.

The steps for training the DBN are as follows:

1. Unroll the DBN into $N$ time slices where $N$ is the length of the evidence $e$;

2. Calculate the distribution over all hidden variables using the forward-backward algorithm;

3. Recalculate the prior probability, transition model and observation model;

(a) The prior probability is based on the value state at $t=0$ and the distribution at $t=0$; 
(b) The transition model is recalculated based on the probability that the past state was $S_{i}$ and the current state is $S_{j}$;

(c) The observation model is calculated based on the probability that the output is $Y$ given the state was $S$.

4. Repeat from step 2 for $M$ number of iterations or until the difference in log likelihood is less then a threshold

EM is an approach used for estimating the hidden states of a DBN from observed evidence. It is used by Ontañón et al. [1] for learning non-reactive behaviour from observation. In the next section, we shift to the general framework of the approach used by Michael Floyd [7], which is Case-based reasoning.

\subsection{Case-Based Reasoning}

Case-Based Reasoning (CBR) is an approach to solve problems by reusing information and knowledge of solutions of a similar problem [13]. Solutions to problems are retrieved from a knowledge base, and learning is done by updating the knowledge base with new solutions. This allows learning in CBR to be incremental and sustained.

Because CBR solves problems using cases, CBR is highly dependent on the quality of the knowledge being represented. In the application of CBR to LFO, Michael Floyd [7] proposes a simplified representation of a case by mapping the sensory input $S$ to the action $A$ at time $t$. 


$$
C_{t}=<S_{t}, A_{t}>
$$

Where $S_{t}$ can in turn be broken down into a set of observable features as reported by various sensors.

Note that in this representation, past inputs and actions are not represented, and so this representation is only suitable for learning reactive behaviour. In Section 3.1 we see how Floyd [7] expands on this representation to include non-reactive behaviour.

Cases are stored in a collection that forms the knowledge base, and the collection is termed a case base. The case base represents the past experiences learned. 


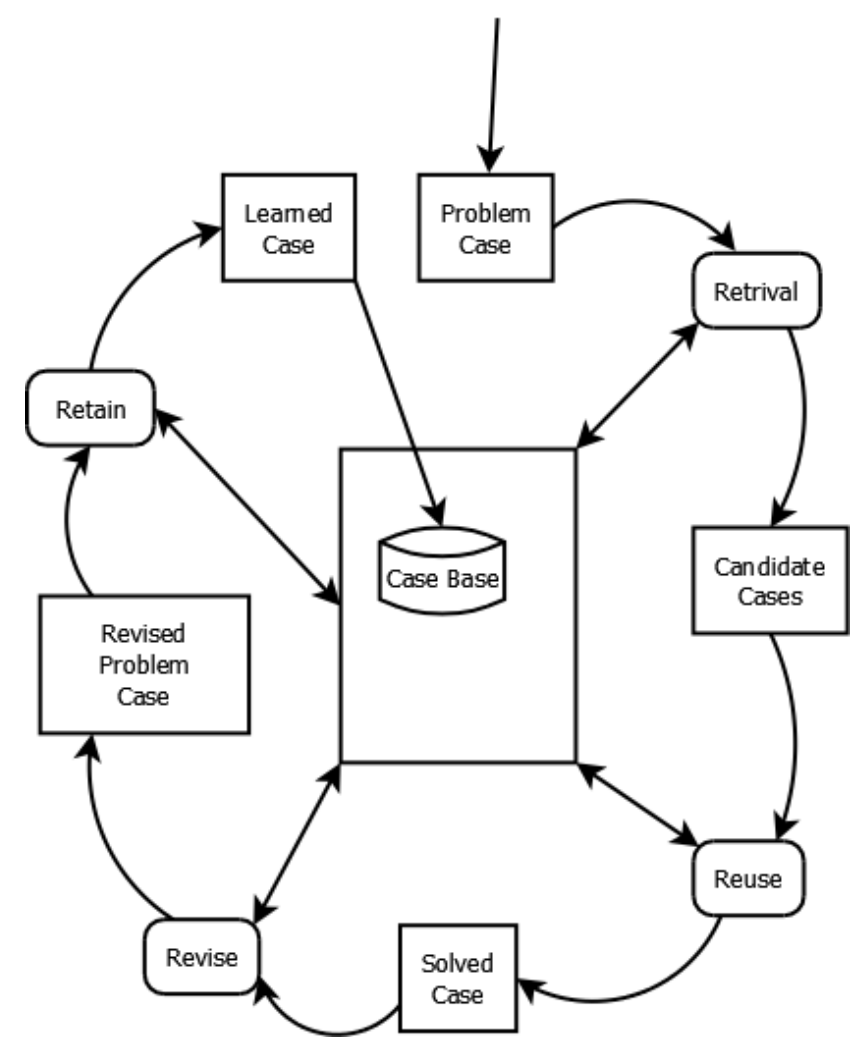

Figure 7: A visual representation of the four-step cycle of case-based reasoning

Reasoning using cases is accomplished through a four-step cycle:

1. Retrieval: Prior to the retrieval step, a problem case is created using the input problem. During the retrieval step, the problem case is compared with all cases in the case base using a domain-specific metric. A non-empty set of the most similar cases is selected from the case base. The number of cases selected during the retrieval step can be constant or variable and is dependent on the approach used.

2. Reuse: During the reuse step, the cases that were selected from the case base in the retrieval step are used to solve the problem case. A solution to the problem 
case is created, either by reusing a solution of a previous case or forming a novel solution by combining solutions of previous cases.

3. Revise: The revise step seeks to correct any errors in the proposed solution during the reuse step. This step can occur only if the CBR receives direct feedback on the proposed solution case. A new modified solution is created by altering the previous solution, and the new solution replaces the old solution.

4. Retain: During the retain step, the correct solution is added back to the case base, to be reused for future problems.

In Section 3.1 we will see how the Temporal Backtracking approach makes use of the CBR for learning non-reactive behaviour.

\subsection{Discussion}

In this section, we looked at the two types of behaviour: reactive and non-reactive. We discussed how an agent with reactive behaviour makes decision based on the most current environment state, whereas an agent with non-reactive behaviour makes decisions based on the state of the environment and its own internal state.

In addition, we looked briefly at a graphical model, the Bayesian network, and at how it can be modelled to learn reactive behaviour. We also looked at how the dynamic Bayesian network can be modelled to learn non-reactive behaviour. We briefly discussed case-based reasoning and introduced how environment state and actions are modelled within the CBR framework. 
In the next section we will look at how previous works has applied Bayesian networks, DBN and CBR to LFO. 


\section{Chapter 3}

\section{State of the Art}

\subsection{Temporal Backtracking}

Michael Floyd proposed the Temporal Backtracking algorithm (TB)in [7] as a solution for LFO of state-based behaviour using CBR. He highlighted that the definition of a case, described in Section 2.5, was insufficient for extracting the temporal relationship between inputs and actions to approximate the behaviour of an agent.

Previously we discussed how a run can encapsulate the behaviour of an agent. A run can be modelled in the following way, with $e_{i}$ being the environment state and $a_{i}$ being the agent's action in response to environment state $e_{i}$ :

$$
r: e_{0} \stackrel{a_{0}}{\rightarrow} e_{1} \stackrel{a_{1}}{\rightarrow} e_{2} \stackrel{a_{2}}{\rightarrow} e_{3} \stackrel{a_{3}}{\rightarrow} \ldots \stackrel{a_{n-2}}{\rightarrow} e_{n-1} \stackrel{a_{n-1}}{\rightarrow} e_{n}
$$

Floyd's proposed solution is to model the case as follows:

$$
C=<e_{0} \stackrel{a_{0}}{\rightarrow} \ldots \stackrel{a_{n-1}}{\rightarrow} e_{n}, a_{n}>
$$

In this model, the sensory input is all the past environment states and action, and is therefore a superset of the history that the agent may use for its internal memory. 
In addition, if each environment state and action pair is a case and a subset of a run is also considered a run, we can simplify the model as such:

$$
C_{t}=<C_{t-1}, e_{t}, a_{t}>
$$

We will also introduce some of the terminology to be used when discussing runs and the TB algorithm. To this end, we define $r(k), r^{i}(k)$ and $r^{a}(k)$, where $k$ denotes the $k$ cases into the past from the current case, $i$ denotes that a input is being returned, $a$ denotes an action that is being returned, and $c$ denotes the case that is being returned.

For example, consider the following run:

$$
r_{1}: a \stackrel{u}{\rightarrow} b \stackrel{w}{\rightarrow} c \stackrel{x}{\rightarrow} d \stackrel{y}{\rightarrow} e \stackrel{z}{\rightarrow}
$$

The current action of $r_{1}$ or $r_{1}^{a}(0)$ would be $z$. The current input of $r_{1}$ or $r_{1}^{i}(0)$ would be $e$. The third input in the past or $r_{1}^{i}(3)$ would be $b$. Lastly, the second-last case in the past or $r_{1}(3)$ would be $b \stackrel{w}{\rightarrow}$.

Because we have redefined a case, the retrieval of cases also has to be redefined. The reuse, revise and retain step of CBR will also need to be updated due to the new definition of a case, but they are not the main focus of this thesis and will not be covered.

The retrieval step now involves selecting runs from the case base. We will call the runs selected from the case base candidate runs. Candidate runs are selected using a $k$ nearest neighbour $(\mathrm{kNN})$ approach. Consider that we have the following problem run $r_{p}: a \stackrel{?}{\rightarrow}$ and we want to select two $(k=2)$ candidate cases from the case base. Consider a case base that consists of the following run: 


$$
r_{1}: a \stackrel{w}{\rightarrow} b \stackrel{x}{\rightarrow} a \stackrel{y}{\rightarrow} c \stackrel{z}{\rightarrow}
$$

For this example, we assume that the kNN algorithm uses the following similarity function when comparing actions and inputs:

$$
\operatorname{sim}(x, y)= \begin{cases}1 & , x=y \\ 0 & , \text { otherwise }\end{cases}
$$

The kNN algorithm will compare the current input of the problem run to each input in the case base. After it does this comparison, it finds two inputs, $r_{b}^{i}(0)$ and $r_{b}^{i}(3)$. Using the inputs, it generates candidate runs by taking the selected inputs and all cases previous to those inputs. This results in the following candidate runs:

- $r_{1}: a \stackrel{w}{\rightarrow}$

$$
\text { - } r_{2}: a \stackrel{w}{\rightarrow} b \stackrel{x}{\rightarrow} a \stackrel{y}{\rightarrow}
$$

After the candidate runs are selected, the TB algorithm eliminates candidate runs until one solution has been found. The solution is assumed to be determined when all remaining candidate runs have the same final action. The pseudocode of the algorithm is shown below. 
Input : current run (run), candidate runs (pastRuns), time offset (time)

Output: action to perform (action)

1 Function stateRetrieve(run, pastRuns, time) : action

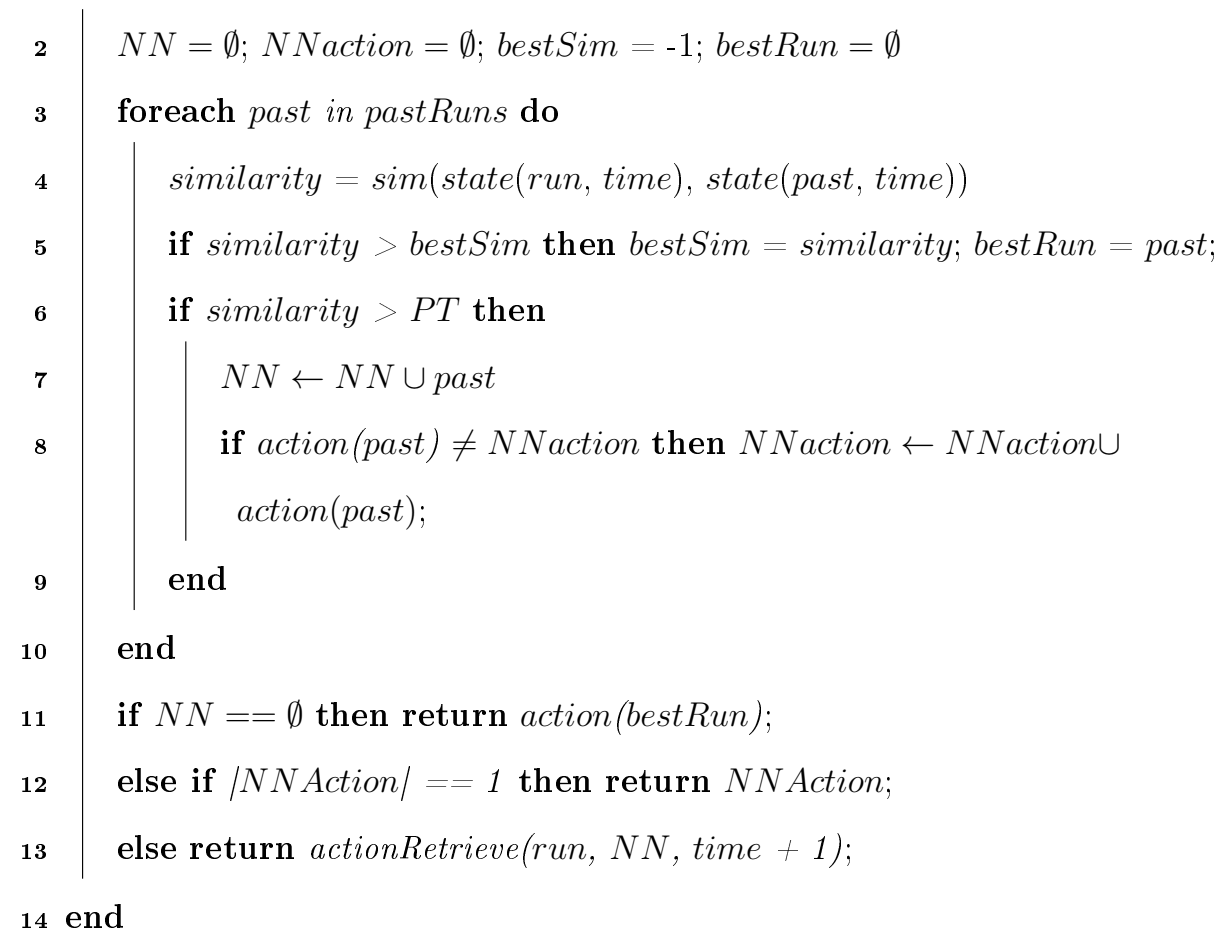

1 Function actionRetrieve(run, pastRuns, time) : action

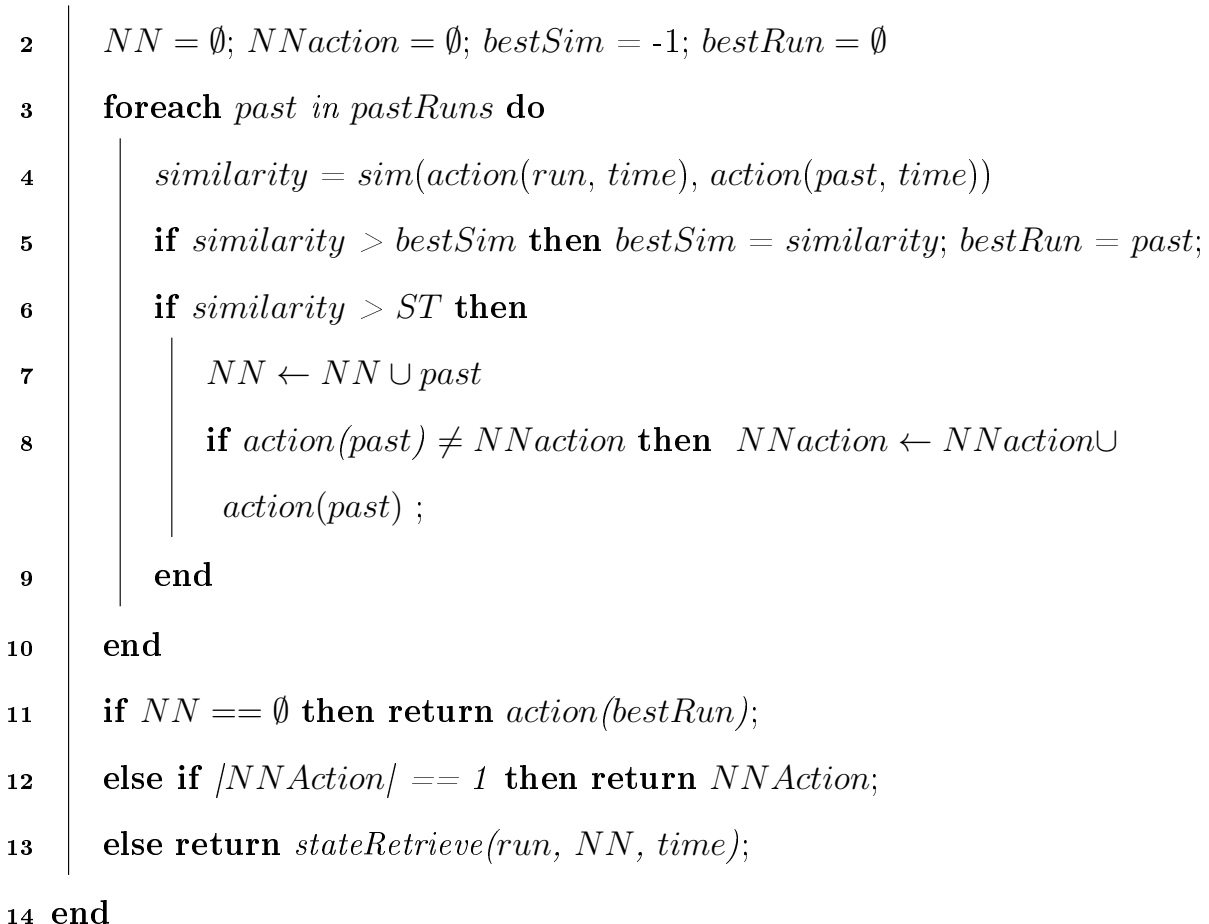

Algorithm 1: The Temporal Backtracking algorithm. PT is defined as the problem threshold and ST is defined as the solution threshold. 
To further explain how the algorithm works, we will use the vacuum robot example from earlier. Consider past runs $r_{1}:$ Space $\stackrel{\text { Forward }}{\rightarrow}$ wall $\stackrel{\text { right }}{\rightarrow}$, and $r_{2}:$ Space $\stackrel{\text { Forward }}{\rightarrow}$ wall $\stackrel{\text { left }}{\rightarrow}$. Now consider we have the following problem run:

$$
r_{p}: \text { Space } \stackrel{\text { Forward }}{\rightarrow} \text { wall } \stackrel{?}{\rightarrow}
$$

The algorithm above introduces the values $P T$ and $S T$, which are the problem threshold and the solution threshold. PT and ST are values used for determining whether states and actions are similar. If a similarity value is lower than the threshold, that state or action is deemed not similar and the run that the state or action is associated with is eliminated. For the vacuum example, similar states or actions will have a similarity of 1.0, and non similar states or actions will have similarity of 0.0 and the value of $P T$ and $S T$ will be set to 0.5 .

Each of the candidate runs $r_{1}$ and $r_{2}$ has a different final action, and thus there is no consensus between those actions. Therefore, we need to use the TB algorithm to eliminate one of the candidate runs. The first step of the TB is comparing the most recent environment state, which is the wall state. Because all candidate runs also have wall as their most recent environment state, they both are above the threshold, and so the TB has to move back to the previous action. When we look at the previous action, we see that the second candidate run has a different action from the problem run. This means that the similarity of that action is below the threshold and the second candidate run is eliminated. This leaves the first candidate run, and thus there is consensus in the final actions of the remaining candidate runs.

There are a number of problems with the TB algorithm shown in Algorithm 1. The first problem is that the behaviour of the algorithm is undefined if the TB 
algorithm fails to reach consensus when it reaches the end of the problem run. The second problem is that the ability to handle runs of variable lengths is undefined. In the algorithm, comparisons are made based on offset from the current case, and runs longer or shorter could lead to cases where the case being compared to is farther in the past than the length of a run. The last problem is that the TB algorithm eliminates candidate runs when the first similarity is below the threshold. Candidate runs are eliminated when the similarity of a past action or input is below a given threshold and the algorithm ignores the rest of the run. This could lead to a situation where very similar runs are eliminated due to an action or input early in the run that has a low similarity.

We will revisit these issues in Section 4.3. In the next section, we will look at how DBN has been used for LFO.

\subsection{Dynamic Bayesian Network}

Ontañón et al. propose that LFO could be accomplished by using a DBN [1]. The authors classified all agents' internal states into three categories. The first category is that agents have no internal state; the second is that an agent's internal state depends on the last $k$ observations; and the third is that an agent's state is not limited to the last $k$ observations, but by any $k$ observations at any point in time. An example of an agent from the first category would be a floor-cleaning robot that is designed to always turn left when it runs into a object. An example of an agent from the second category would be an agent driving on a highway that needs to remember a road sign that it saw $k$ observations ago. Lastly, an example of an agent 
from the third category is a surgeon who uses their previous experiences to make crucial decisions when performing surgery.

The experiment by Ontañón et al. [1] was designed to test the accuracy of a DBN against other learning algorithms that are suited for each category. For category one, Bayesian networks $(\mathrm{BN})$ and neural networks $(\mathrm{NN})$ were chosen. For category two, the sliding window method mentioned in the previous chapter for both BN and NN were chosen. Both BN and NN were modelled to use the previous observation, or $k=2$. Lastly, from category three, the DBN and Input Output Hidden Markov Model (IOHMM) were chosen. The model for DBN is provided in Section 2.3. The model for IOHMM is similar to the DBN model, except that the hidden state, $S_{t}$ depends only on the input $X_{t}$ and the previous hidden state, $S_{t-1}$, and does not depend on the previous action $Y_{t-1}$. All the graphical models used in the experiment were supervised.

The algorithms were tasked with learning the behaviours of 5 floor-cleaning agents. The agent perceives the environment through eight binary sensors: four object sensors and four range sensors. The object sensor detects whether the closest object is a dirt object or a wall and will return True if the closest object is a dirt object. The range sensor detects whether there is an object adjacent to the agent and will return True if the object is adjacent. An object and a range sensor are placed on each side of the agent; the configuration of these sensors is shown in Figure 8. Information from the sensors can be represented as an 8-tuple, which means that the perception of the agent can take one of 256 different states. Figure 9 shows an example of an agent with the corresponding sensory tuple. The agent can take one of five different actions: UP, DOWN,LEFT, RIGHT, and STAND. The STAND 


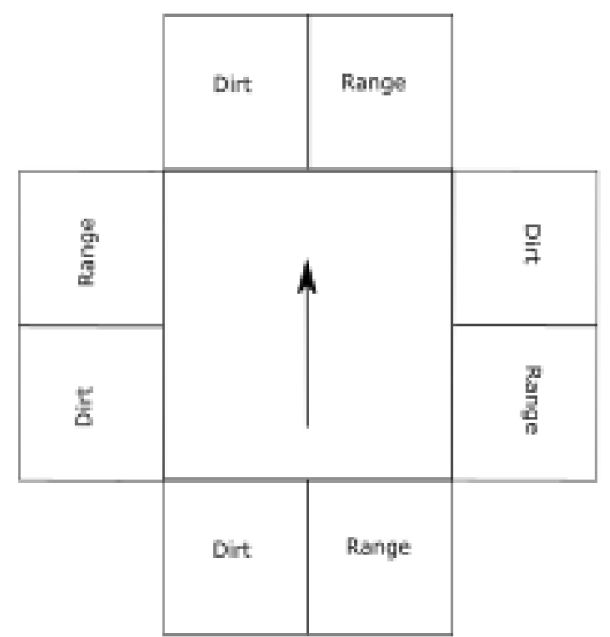

$\left[\mathrm{N}_{D}, \mathrm{~N}_{\mathrm{R}}, \mathrm{E}_{\mathrm{D}}, \mathrm{E}_{\mathrm{R}}, \mathrm{S}_{\mathrm{D}}, \mathrm{S}_{\mathrm{R}}, \mathrm{W}_{\mathrm{D}}, \mathrm{W}_{\mathrm{R}}\right]$

Figure 8: Shown on the left is the configuration of the eight sensors, four dirt and four range, used with the vacuum robot. Shown on the right is a tuple representing the binary information from each of the sensors. $N_{D}$ represents the north dirt sensor and $N_{R}$ represents the north range sensor.

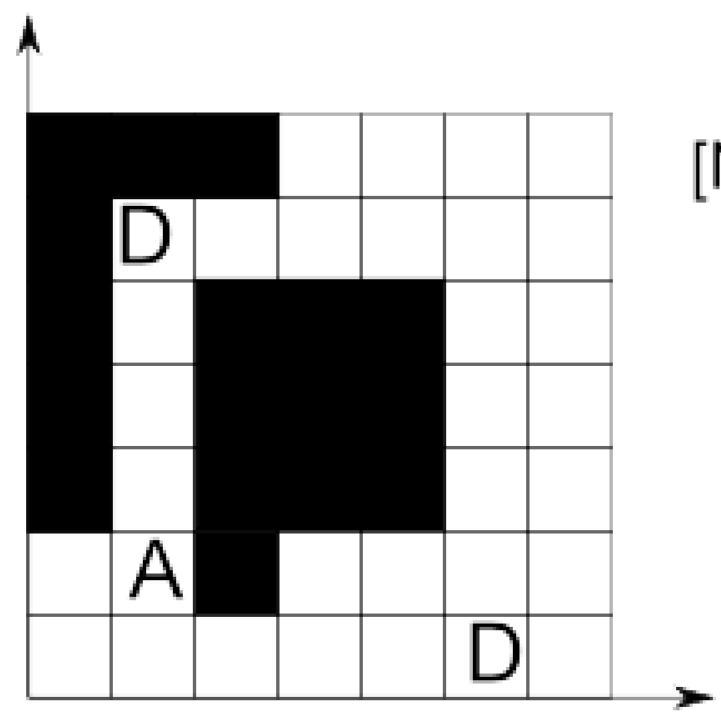

$$
\begin{gathered}
{\left[N_{D}, N_{R}, E_{D}, E_{R}, S_{D}, S_{R}, W_{D}, W_{R}\right]} \\
{[T, F, F, T, F, F, F, F]}
\end{gathered}
$$

Figure 9: The agent (A), dirt (D) and an obstruction (opaque squares). Shown on the right is the corresponding sensory tuple, demonstrating the sensory data from each of the eight sensors. 
action means that the agent will not move in any direction from its current location. The 5 floor-cleaning agents were:

Smart Random This agent will randomly choose the direction of travel, but will move toward any dirt patch it sees;

Smart Straight Line The agent will pick a random direction and move in that direction until it hits an obstacle; it will then repeat, but as with the smart random, will move toward any dirt it sees;

Zig Zag The agent will move straight to the right until it hits an obstacle, then move down until it hits an obstacle, then move right until it hits an obstacle, and keep repeating this behaviour;

Fixed Sequence The agent moves in a fixed sequence;

Smart Explorer The agent will randomly pick a move toward an unexplored square. It keeps in its memory which squares it has traversed. As with the Smart Random and Smart Straight Line agents, this agent will move towards dirt if it sees it. Once it has visited each location it will take the STAND action.

The traces used to train each of the learning algorithms were composed of each agent running 1000 steps in 7 different maps. The maps were either of $8 \times 8$ size or $32 \times 32$ in size with anywhere between 2 to 7 dirt squares located at predetermined locations on the map. In addition to dirt objects a number of obstacles were added into the map at predetermined locations. 


\begin{tabular}{|c|c|c|c|c|c|c|}
\hline & NN & BN & NNk2 & BNk2 & IOHMM & DBN \\
\hline \hline Smart Random & 32.0 & 30.9 & 32.0 & 31.0 & 31.0 & 31.1 \\
\hline Smart Straight Line & 40.0 & 40.7 & 85.1 & 84.8 & 77.2 & 84.3 \\
\hline Zig-Zag & 41.3 & 40.9 & 73.7 & 91.6 & 65.2 & 83.4 \\
\hline Fixed Sequence & 43.2 & 36.2 & 66.4 & 51.9 & 85.8 & 88.2 \\
\hline Smart Explorer & 48.4 & 49.3 & 79.1 & 77.6 & 65.3 & 79.3 \\
\hline Average & 41.0 & 39.6 & 67.26 & 67.38 & 64.9 & 72.3 \\
\hline
\end{tabular}

Table 1: Original results [1] showing the accuracy of Bayesian network (BN), neural network $(\mathrm{NN})$, sliding window version of $\mathrm{BN}$ and $\mathrm{NN}$ where $k=2(\mathrm{BNk} 2$ and NNk2), input-output hidden markov model (IOHMM), and dynamic Bayesian network (DBN)

We notice that none of the graphical models were able to learn the Smart Random behaviour.

Ontañón et al were able to draw two conclusions. The first was that supervised learning approaches cannot offer a general solution for LFO. The supervised learning approaches were able to learn the behaviours of some of the agents, but not all.

Their second conclusion was that to learn behaviours when an agent has a finite discrete internal state, a different learning approach is needed. The DBN algorithm accounts for the internal state by representing it as a hidden variable.

There are a number of problems with the results presented by the authors. The first problem is that various mean accuracies were reported without the standard deviation. Without the error rates, there is no way of knowing how reliable the accuracies are, especially since there is a great deal of randomness in the agent 
behaviours to be learned. This problem can be remedied by running the experiment multiple times to calculate an average mean and standard deviation. The second problem is that accuracy was used instead of the F-measure. This is problematic in the presence of unbalanced data, in this case, the skew in the action selected by the behaviours to be learned. This problem can be remedied by calculating a Macro-F1 [14]. A Macro-F1 remedies the class imbalance problem by emphasizing the performance of rare actions and is obtained by averaging the F1 score of each action. The F1 score is a harmonic mean of the precision and recall and is combined in the following way:

$$
\begin{gathered}
\text { Recall }=\frac{\text { number of correct actions predictions }}{\text { number of action examples }} \\
\text { Precision }=\frac{\text { number of correct actions predictions }}{\text { number of action predictions }} \\
F 1=\frac{2 \times \text { Recall } \times \text { Precision }}{\text { Recall }+ \text { Precision }}
\end{gathered}
$$

\subsection{Discussion}

In this chapter we looked at a CBR solution through the TB algorithm and a graphical model solution for LFO. Issues with the implementation of both those solutions were discussed.

The TB algorithm has a number of undefined behaviours. These behaviours consist of failing to reach consensus, comparisons of non-equal candidate and problem runs, and issues with eliminating candidate runs based on the first instance where the similarity value is below the threshold. For the graphical model solution, the 
results presented by the authors had a great deal of uncertainty because errors were not provided and accuracy was used instead of the F-measure.

The goal of the next chapter will be to replicate the experiment for the stochastic solution to resolve some of the uncertainty. In addition, a comparative analysis will be conducted between the CBR and the stochastic solution using the cleaning robot as a benchmark. Lastly we will analyze the undefined behaviours with the goal of defining them. 


\section{Chapter 4}

\section{Analysis of Existing Approaches}

In the previous chapter, we looked at two different solutions for LFO for non-reactive behaviours. We discussed a CBR approach that has redefined cases to allow for behavioural information to be captured. We noted that the proposed TB algorithm leaves some situations undefined. In addition, we discussed a graphical model solution that uses a hidden state to infer the internal behaviour of an agent, but the experimental results were lacking.

In this chapter, we will look to resolve some of these problems by replicating the experiment done for DBN. The experiment will then be repeated for the TB algorithm and the results will be compared with the results of the DBN experiment. Lastly, we will analyze the situation where the undefined behaviour mentioned in the previous chapter can occur. 


\subsection{Learning from Observation using a Dynamic Bayesian Network}

In the original results [1], DBN had a higher overall average accuracy than all the other learning techniques. To verify the results, the experiment was retested under the same conditions.

The code used to perform the experiments was given by the authors of the original results. As with the original experiment, the graphical models was tested using a supervised dataset. A dataset consisted of 7 traces for each of the 5 agents with each trace being 1000 steps long, and a trace being the agent's behaviour in one of the 7 different maps used. The traces used in the original experiment were not provided by the authors and had to be generated by simulating the agent in the map and recording the agents behaviour for 1000 steps. Testing of each agent was done using 7 -fold cross validation with each fold being a trace. To address the issue with standard deviations missing, the original results were repeated six times using six different datasets. In addition, six datasets were used due to time constraints and to limit the total experiment time to one week.

The total time to run the experiment on the six datasets took about six days to complete. The experiments were run on a dedicated machine consisting of an Intel i7 processor with $12 \mathrm{~GB}$ of RAM. For both DBN and IOHMM, the internal state was limited to 4 different values and both were trained using the EM algorithm for 20 iterations.

In the original results, the mean accuracy of each of the learning techniques for each of the behaviours were provided, but a standard error was not provided with 
the mean.

\begin{tabular}{|c|c|c|c|}
\hline Retested Behaviour & NN & $\mathrm{BN}$ & NNk2 \\
\hline Smart Random & $34.70 \pm 1.04$ & $33.89 \pm 0.99$ & $34.57 \pm 0.35$ \\
\hline Smart Straight Line & $50.58 \pm 0.80$ & $50.12 \pm 0.48$ & $93.81 \pm 0.37$ \\
\hline Zig-Zag & $51.38 \pm 0.33$ & $47.97 \pm 0.53$ & $95.40 \pm 0.18$ \\
\hline Fixed Sequence & $53.21 \pm 1.26$ & $40.51 \pm 0.40$ & $66.68 \pm 1.81$ \\
\hline Smart Explorer & $34.19 \pm 17.63$ & $31.49 \pm 16.14$ & $75.63 \pm 5.40$ \\
\hline Average & 44.81 & 40.80 & 73.22 \\
\hline
\end{tabular}

\begin{tabular}{|c|c|c|c|}
\hline Retested Behaviour & BNk2 & IOHMM & DBN \\
\hline \hline Smart Random & $33.50 \pm 0.56$ & $34.50 \pm 0.82$ & $34.54 \pm 0.59$ \\
\hline Smart Straight Line & $\mathbf{9 1 . 3 7} \pm \mathbf{0 . 7 6}$ & $\mathbf{6 7 . 0 7} \pm \mathbf{3 . 7 9}$ & $\mathbf{7 0 . 4 2} \pm \mathbf{1 . 3 3}$ \\
\hline Zig-Zag & $\mathbf{8 7 . 9 7} \pm \mathbf{0 . 2 7}$ & $\mathbf{7 1 . 0 4} \pm \mathbf{4 . 2 1}$ & $\mathbf{7 8 . 6 3} \pm \mathbf{1 . 4 0}$ \\
\hline Fixed Sequence & $\mathbf{4 7 . 0 8} \pm \mathbf{0 . 3 1}$ & $\mathbf{4 4 . 5 1} \pm \mathbf{1 . 1 6}$ & $\mathbf{4 4 . 2 2} \pm \mathbf{1 . 0 3}$ \\
\hline Smart Explorer & $\mathbf{4 3 . 2 5} \pm \mathbf{1 2 . 1 4}$ & $\mathbf{2 9 . 9 4} \pm \mathbf{1 5 . 6 6}$ & $\mathbf{3 6 . 1 2} \pm \mathbf{1 4 . 3 5}$ \\
\hline Average & 60.64 & 49.41 & 52.79 \\
\hline
\end{tabular}

Table 2: A comparison of the original accuracies shown in the top table [1] versus the retested results in the bottom two tables. The tables show the values of Bayesian network (BN), neural network (NN), sliding window version of $\mathrm{BN}$ and $\mathrm{NN}$ where $k=2(\mathrm{BNk} 2$ and $\mathrm{NNk} 2)$, input-output hidden markov model (IOHMM), and dynamic Bayesian network (DBN). The experiment was done using 7-fold cross validation, where each fold is 1000 steps long; this was repeated for six different datasets. Bold values are either above or below their original values if we factor in the margin of error 
We can see that most of the retested values, shown in bold, are different from their original values if we factor in the margin of error. The largest change among the retested values was the Smart Straight Line and Zig-Zag behaviour. With regard to the Smart Straight Line behaviour, the biggest discrepancy between the original behaviour and the retested behaviour is with NN and DBN, with NN showing in increase of $\sim 10$ percentage points and the DBN showing a decrease of $\sim 14$ percentage points. With regard to the Zig-Zag behaviour, the biggest discrepancy between the original behaviour and the retested behaviour is with NNk2 and IOHMM, with NNk2 showing an increase of $\sim 22$ percentage points and IOHMM showing a decrease of $\sim 7$ percentage points.

If we compare the original results to the retested results we see that most of the values are outside a $95 \%$ confidence interval. This leads us to believe that randomness is not the principle cause of the difference. There are a couple of possible explanations for this difference. The first possible explanation is that there are bugs in the code that generated the original results or there is a bug in the code that generated the retested results. Another possible explanation is that the code could have changed. The authors of the original paper wrote a follow-up paper [6] and the code that was received from the authors had test from the follow-up paper. When creating test for the follow-up paper they authors could have change the code to fit the needs of the new paper. 


\begin{tabular}{|c|c|c|c|}
\hline Retested Behaviour & NN & BN & NNk2 \\
\hline \hline Smart Random & $34.07 \pm 0.54$ & $26.84 \pm 0.28$ & $34.55 \pm 0.11$ \\
\hline Smart Straight Line & $50.31 \pm 0.25$ & $40.04 \pm 0.25$ & $93.78 \pm 0.06$ \\
\hline Zig-Zag & $36.05 \pm 0.15$ & $33.47 \pm 0.11$ & $86.73 \pm 0.29$ \\
\hline Fixed Sequence & $48.52 \pm 1.05$ & $33.00 \pm 0.08$ & $65.78 \pm 0.71$ \\
\hline Smart Explorer & $25.61 \pm 3.64$ & $23.69 \pm 4.54$ & $52.30 \pm 1.56$ \\
\hline Average & 38.91 & 31.41 & 66.63 \\
\hline
\end{tabular}

\begin{tabular}{|c|c|c|c|}
\hline Retested Behaviour & BNk2 & IOHMM & DBN \\
\hline \hline Smart Random & $26.80 \pm 0.23$ & $27.56 \pm 0.18$ & $27.72 \pm 0.22$ \\
\hline Smart Straight Line & $73.47 \pm 0.16$ & $53.81 \pm 0.60$ & $56.70 \pm 0.40$ \\
\hline Zig-Zag & $62.97 \pm 0.12$ & $47.94 \pm 0.55$ & $52.64 \pm 0.88$ \\
\hline Fixed Sequence & $39.20 \pm 0.16$ & $36.44 \pm 0.57$ & $35.85 \pm 0.21$ \\
\hline Smart Explorer & $31.99 \pm 3.52$ & $24.88 \pm 3.97$ & $26.50 \pm 4.88$ \\
\hline Average & 46.89 & 38.13 & 39.88 \\
\hline
\end{tabular}

Table 3: Average F1 measure of the retested results. The tables show the values of Bayesian network (BN), neural network (NN), sliding window version of BN and NN where $k=2(\mathrm{BNk} 2$ and NNk2), input-output hidden markov model (IOHMM), and dynamic Bayesian network (DBN).

In Table 3, we can see that NNk2 and NN have a higher F1-measure than all the other graphical model solutions for the Smart Random behaviour. This is due to the STAND action being selected instead of the other four actions. One possible explanation is the training of the graphical model has not eliminated the probability 
of the STAND action appearing. This can be the result of either one or a combination of variability of weight initialization, EM iterations, or not enough training data. The problem of the STAND action appearing when it should not be also extends to the other behaviours with the exception of Smart Explorer.

\begin{tabular}{|c|c|c|c|}
\hline Retested Train Time & NN & BN & NNk2 \\
\hline \hline Smart Random & $4.09 \pm 1.07$ & $0.15 \pm 0.02$ & $4.93 \pm 0.83$ \\
\hline Smart Straight Line & $9.77 \pm 4.75$ & $0.19 \pm 0.02$ & $7.30 \pm 1.35$ \\
\hline Zig-Zag & $5.02 \pm 2.21$ & $0.17 \pm 0.03$ & $9.09 \pm 5.25$ \\
\hline Fixed Sequence & $5.02 \pm 2.21$ & $0.13 \pm 0.02$ & $6.41 \pm 1.48$ \\
\hline Smart Explorer & $4.91 \pm 1.88$ & $0.13 \pm 0.01$ & $5.15 \pm 0.59$ \\
\hline Average & 5.76 & 0.15 & 6.58 \\
\hline
\end{tabular}

\begin{tabular}{|c|c|c|c|}
\hline Retested Train Time & BNk2 & IOHMM & DBN \\
\hline \hline Smart Random & $0.33 \pm 0.04$ & $150.56 \pm 0.65$ & $148.06 \pm 0.59$ \\
\hline Smart Straight Line & $0.34 \pm 0.03$ & $702.71 \pm 182.71$ & $433.86 \pm 44.40$ \\
\hline Zig-Zag & $0.33 \pm 0.02$ & $912.05 \pm 132.75$ & $458.42 \pm 37.95$ \\
\hline Fixed Sequence & $0.32 \pm 0.02$ & $892.84 \pm 71.02$ & $862.57 \pm 104.24$ \\
\hline Smart Explorer & $0.33 \pm 0.02$ & $679.96 \pm 124.14$ & $550.97 \pm 91.03$ \\
\hline Average & 0.33 & 667.62 & 490.78 \\
\hline
\end{tabular}

Table 4: The average time in seconds needed to train the graphical network (Bayesian network $(\mathrm{BN})$, neural network $(\mathrm{NN})$, sliding window version of $\mathrm{BN}$ and NN where $k=2$ (BNk2 and NNk2), input-output hidden markov model (IOHMM), and dynamic Bayesian network (DBN)) 
If we look at Table 4 we can see that IOHMM has the overall average training time at 667.62 seconds or about 11 minutes. We can also see that $\mathrm{BN}$ and $\mathrm{BN}$ have the lowest training time out of all the other graphical models. This is because they have the least amount of nodes to train. DBN has the most nodes of all the graphical models used, but it does not have the longest training time. One possible explanation for this is that the hidden state is dependent on the previous action whereas the hidden state of the IOHMM is not. Most of the agent's behaviours rely on the previous action to decide the next action. Because the hidden state in the IOHMM is not dependent on the previous action, the IOHMM need to be trained more to replicate this dependency. 


\begin{tabular}{|c|c|c|c|}
\hline Retested Run Time & NN & BN & NNk2 \\
\hline \hline Smart Random & $5.71 \pm 0.11$ & $12.49 \pm 0.60$ & $5.99 \pm 0.06$ \\
\hline Smart Straight Line & $5.68 \pm 0.06$ & $12.16 \pm 0.05$ & $6.49 \pm 0.64$ \\
\hline Zig-Zag & $5.62 \pm 0.03$ & $12.19 \pm 0.04$ & $5.93 \pm 0.02$ \\
\hline Fixed Sequence & $5.70 \pm 0.09$ & $12.23 \pm 0.07$ & $5.92 \pm 0.02$ \\
\hline Smart Explorer & $5.49 \pm 0.03$ & $12.20 \pm 0.05$ & $5.57 \pm 0.01$ \\
\hline Average & 5.64 & 12.25 & 5.98 \\
\hline
\end{tabular}

\begin{tabular}{|c|c|c|c|}
\hline Retested Run Time & BNk2 & IOHMM & DBN \\
\hline \hline Smart Random & $20.70 \pm 0.25$ & $<0.01 \pm 0.00$ & $<0.01 \pm 0.01$ \\
\hline Smart Straight Line & $20.73 \pm 0.04$ & $<0.01 \pm 0.00$ & $<0.01 \pm 0.01$ \\
\hline Zig-Zag & $20.70 \pm 0.02$ & $<0.01 \pm 0.01$ & $<0.01 \pm 0.01$ \\
\hline Fixed Sequence & $20.76 \pm 0.11$ & $<0.01 \pm 0.01$ & $<0.01 \pm 0.00$ \\
\hline Smart Explorer & $20.70 \pm 0.03$ & $<0.01 \pm 0.01$ & $<0.01 \pm 0.01$ \\
\hline Average & 20.72 & $<0.01$ & $<0.01$ \\
\hline
\end{tabular}

Table 5: The average time in milliseconds that the graphical network (Bayesian network $(\mathrm{BN})$, neural network $(\mathrm{NN})$, sliding window version of $\mathrm{BN}$ and $\mathrm{NN}$ where $k=2$ (BNk2 and NNk2), input-output hidden markov model (IOHMM), and dynamic Bayesian network (DBN)) take to predict the action once they have respectively received the environment state

The next thing that we will look at is the run time of each of the graphical models. We define the run time as the time each model takes to predict the next action when given the current state of the environment. The run time of each graphical model 
is shown in Table 5. In IOHMM and DBN the run times are all $<0.01$ and are significantly lower than the other graphical models. This at first is surprising, given the complexity of those algorithms. The reason why the run time values are low is because the IOHMM and DBN were programmed using a different language. The training of all the graphical models and the programing of the NN, NNk2, BN and BNk2 models were all programmed with Matlab using the Bayesian Network Toolkit package. The testing of IOHMM and DBN were programmed using Java. As such, we cannot accurately compare the run times of DBN and IOHMM with the rest of the graphical models. When we look at the models programmed with Matlab we see that BNk2 has the highest run time and is significantly higher than the other graphical models. This could be due to the implementation of the BNk2 model.

From the retested results we can say that NNk2 is better than DBN. This is shown clearly when comparing accuracies and F1-measures. One possible reason for NNk2 performing better than DBN is due to the training of the DBN and a small training set. Future work should include retesting all graphical models with a larger training set, and for training of IOHMM and DBN with EM to have more iterations and a smaller threshold.

\subsection{TB to Infer Internal State}

In this section, we will test the TB algorithm on the same benchmark as in Section 4.1 so we can compare the results side-by-side and draw some conclusions. We will look at the configurations of the TB algorithm for running the same benchmark as Section 4.1. The key configuration that this section will focus on is the similarity 
metric used for actions and inputs.

The similarity of a case is broken down into similarity of an action and similarity of an input. The agent can select one of five possible actions for each input received from the environment. Each of the actions are atomic and non-overlapping. To calculate the similarity of two actions, we will use a binary similarity function, shown in Equation 3. If the actions are equal, we return a similarity value of 1 and 0 otherwise.

$$
\operatorname{sim}\left(a_{1}, a_{2}\right)= \begin{cases}1 & a_{1}=a_{2} \\ 0 & \text { otherwise }\end{cases}
$$

As for the inputs, an input from an agent can be expressed as a binary string $i$ :

$$
i=s_{1} s_{2} s_{3} s_{4} s_{5} s_{6} s_{7} s_{8}
$$

where each $s_{m}$ represents the binary output of sensor $m$. Because each sensor returns a binary value, we will use the following equation to calculate the similarity of a sensor.

$$
\operatorname{sim}\left(s_{1}, s_{2}\right)= \begin{cases}1 & , s_{1}=s_{2} \\ 0 & , \text { otherwise }\end{cases}
$$

Using the example in Figure 9, the input can be represented as $i=T F F T F F F F$. The similarity between inputs can be represented as such:

$$
\operatorname{sim}\left(i_{1}, i_{2}\right)=1-\frac{\sum_{k=1}^{8} \operatorname{sim}\left(i_{1, k}, i_{2, k}\right)}{8}
$$


where $i_{1}$ is the first input, $i_{2}$ is the second input and $i_{1, k}$ is the $k$-th sensor for the first input. From Equation 4, we can see that the similarity for inputs is the average similarity of each of the eight sensors.

The TB algorithm was trained using the same dataset used for the DBN learning algorithms. As with the DBN learning algorithm test, a 7-fold validation was used for each run of the test, with each fold consisting of 1000 data points. The total run time of the experiment was about 15 minutes, with each behaviour taking about 3 minutes. This experiment was run using the same machine that ran the retested DBN experiment.

The validation of actions from the TB learning algorithm was different from the DBN algorithm. In the DBN learning algorithm, both testing and training set originated from an agent's trace. For the TB algorithm, the training set still originated from the agent's trace, but for testing the algorithm was used to predict the action of an agent in the environment and the actions were checked against the agent's action under the same situation. The purpose of this change was to see the behaviour of the algorithm in the environment and see if the algorithm could behave similar to the agent. Because a few of the behaviours were random in nature, the same six datasets used in earlier experiments were used to sufficiently test the algorithm.

The Problem Threshold for comparison of inputs was set to 0.5 and the Solution Threshold for comparison of actions was set to 0 . In addition, multiple values of $k$ (the number of nearest neighbours) were selected to analyze the impact on the performance. Before $k$ candidate runs are selected, potential candidate runs are randomly shuffled to eliminate any possible bias due to ordering. 
In this section, we have discussed some of the key configurations that were used in setting up the TB algorithm. In the following section, we will examine the TB algorithm running the same test as the DBN.

\subsubsection{TB Using Non-deterministic Candidate Run Selection}

From the data in Table 6 , we see that different values of $k$ have a minimal impact on the performance on most of the behaviour except for the Smart Explorer behaviour. 


\begin{tabular}{|c|c|c|c|c|}
\hline TB Accuracy Results & TB k $=4$ & TB k $=10$ & TB k $=20$ & TB k $=100$ \\
\hline \hline Smart Random & $30.82 \pm 0.59$ & $30.63 \pm 0.35$ & $30.45 \pm 0.30$ & $29.91 \pm 0.37$ \\
\hline Smart Straight Line & $88.06 \pm 0.76$ & $93.28 \pm 0.30$ & $92.78 \pm 0.41$ & $89.28 \pm 3.03$ \\
\hline Zig-Zag & $44.07 \pm 1.21$ & $44.53 \pm 1.76$ & $46.01 \pm 0.63$ & $53.43 \pm 1.30$ \\
\hline Fixed Sequence & $25.27 \pm 0.86$ & $25.44 \pm 1.22$ & $25.06 \pm 0.86$ & $26.17 \pm 3.76$ \\
\hline Smart Explorer & $3.40 \pm 1.90$ & $5.50 \pm 6.60$ & $10.04 \pm 19.56$ & $28.49 \pm 25.07$ \\
\hline Average & 33.27 & 39.88 & 40.87 & 45.48 \\
\hline
\end{tabular}

\begin{tabular}{|c|c|c|c|c|}
\hline TB F1 Results & TB k $=4$ & TB k $=10$ & TB k $=20$ & TB k $=100$ \\
\hline \hline Smart Random & $30.78 \pm 0.57$ & $30.62 \pm 0.36$ & $30.43 \pm 0.30$ & $29.90 \pm 0.36$ \\
\hline Smart Straight Line & $42.95 \pm 2.45$ & $50.66 \pm 1.46$ & $48.66 \pm 1.15$ & $44.12 \pm 3.45$ \\
\hline Zig-Zag & $32.83 \pm 0.56$ & $34.78 \pm 0.96$ & $35.40 \pm 0.59$ & $39.86 \pm 0.70$ \\
\hline Fixed Sequence & $24.49 \pm 0.78$ & $24.56 \pm 1.35$ & $23.93 \pm 0.70$ & $25.77 \pm 3.89$ \\
\hline Smart Explorer & $7.60 \pm 2.58$ & $8.14 \pm 4.91$ & $10.20 \pm 12.62$ & $21.20 \pm 14.57$ \\
\hline Average & 26.3 & 27.88 & 27.84 & 29.54 \\
\hline
\end{tabular}

Table 6: Accuracy (Top) and F1-Measure (Bottom) of the temporal backtracking algorithm for $k$ values $4,10,20$, and 100

In the Smart Random behaviour, we see that as the value of $k$ increases the accuracy and F1-measure drop. This drop could be attributed to a combination of factors. Due to the large number of candidate runs, the TB algorithm cannot discriminate between non-similar candidate runs due to a relaxed threshold. For example, consider an reactive agent that will only take the $L E F T$ action when it 
receives the input $s_{1}$ and only takes the RIGHT action when it receives the input $s_{2}$. If the threshold is low enough, the TB algorithm will assume that both inputs are similar. Therefore when the TB algorithm receives input $s_{1}$, and in the candidate runs $s_{1}$ and $s_{2}$ appear, it will assume that there is no consensus instead of eliminating the candidate run with $s_{2}$. This means that with the current threshold values, the TB algorithm cannot correctly discriminate between similar inputs.

The Smart Straight Line behaviour has a minor random component to the behaviour, which entails the agent selecting a random direction to travel in a straight line and select another direction once it has hit a wall. As the agent moves in a straight line, consecutive actions are repeated until it hits a wall. From the high accuracy of the TB algorithm it is possible to conclude that the TB algorithm is able to predict repeated consecutive actions. Although the accuracy is high, it is not perfect. When we compare this to the results from the DBN, we see that it performed better and the accuracy is closer to NNk2 and BNk2 at higher values of $k$.

The NNk2 and BNk2 use the last $k-1$ observation when predicting the next action. For a large duration of the Smart Straight Line behaviour, the agent is travelling in a straight line. This means that while travelling in a straight line, the next action is dependent on a previous action. Learning techniques that take into consideration past observations (NNk2, BNk2, IOHMM, and DBN), perform better than those that do not. This is also true of the TB algorithm. We can see that the performance of the algorithm is better than NN and BN.

The Zig-Zag behaviour performs worse than the Smart Straight Line behaviour. We see that the accuracy of the Smart Straight Line is approximately double the 
accuracy of the Zig-Zag behaviour. One possible explanation for this is the TB algorithm not being able to correctly select between the LEFT and RIGHT actions and a bias between the LEFT and RIGHT actions and the UP and DOWN actions in the training set. This bias causes the TB algorithm to select either LEFT of RIGHT instead of other actions.

The Fixed Sequence behaviour does not have any random component to it and moves in a fixed pattern. From the data, we can see that as the value of $k$ increases, both accuracy and F1-measure of the TB algorithm either does not increase but remains constant, or increases by a negligible amount. One possible explanation for this is that by selecting cases based on the similarity of the current input, candidate runs are selected randomly. Consider a sequence of two action where the actions follow the sequence below:

$$
a_{1} \rightarrow a_{2} \rightarrow a_{1} \rightarrow a_{1} \rightarrow a_{2}
$$

If $s_{m}$ is the current input and we generate a list of candidate runs, a possible list is as follows:

$$
\begin{aligned}
& \text { - } r_{c_{1}}: \ldots s_{2} \stackrel{a_{2}}{\rightarrow} s_{1} \stackrel{a_{1}}{\rightarrow} s_{m} \stackrel{a_{2}}{\rightarrow} \\
& \text { - } r_{c_{2}}: \ldots s_{2} \stackrel{a_{2}}{\rightarrow} s_{1} \stackrel{a_{1}}{\rightarrow} s_{m} \stackrel{a_{1}}{\rightarrow}
\end{aligned}
$$

This example shows that although each of the candidate runs has the same current input, they are in different positions in their sequence. The first candidate run is most likely in the second action of the sequence and the second action is most likely in the second last action of the sequence. Actions that are taken by the robot are independent of the input received from the environment and is shown by both candidate runs having the same inputs, but be in random location of the sequence. 
The Smart Explorer is the most complex of all the behaviours. The behaviour has both short- and long-term goals: the short-term goal of moving toward any dirt it find; and the long-term goal of visiting each location on the map. From the data, we notice that as the value of $k$ increases, the accuracy and F1-measure increases. By increasing the value of $k$, more of the Smart Explorer behaviour is exposed to the TB algorithm and thus the algorithm can better estimate the long-term goal.

In the Smart Explorer behaviour, the standard deviation is significantly high. One possible explanation for the high standard deviation is the randomness of the agent affecting the training set. The Smart Explorer behaviour selects a random location that it has not previously visited and moves in that direction. If there are no valid locations to move to, it will take the STAND action. The randomness of the agent could generate training runs in which the STAND action does not appear, but during testing it could reach a situation where the STAND action is the only one possible. Because the STAND action does not appear in the training runs, the STAND action is never selected and thus could produce a low accuracy. 


\begin{tabular}{|c|c|c|c|c|}
\hline TB Run Time Results & TB $\mathrm{k}=4$ & TB $\mathrm{k}=10$ & TB $\mathrm{k}=20$ & $\mathrm{~TB} \mathrm{k}=100$ \\
\hline \hline Smart Random & $2.88 \pm 0.09$ & $3.02 \pm 0.07$ & $3.10 \pm 0.06$ & $4.19 \pm 0.07$ \\
\hline Smart Straight Line & $3.30 \pm 0.38$ & $3.16 \pm 0.13$ & $3.22 \pm 0.10$ & $4.14 \pm 0.14$ \\
\hline Zig-Zag & $3.15 \pm 0.23$ & $2.96 \pm 0.02$ & $3.48 \pm 0.10$ & $4.31 \pm 0.11$ \\
\hline Fixed Sequence & $3.06 \pm 0.09$ & $2.94 \pm 0.08$ & $3.24 \pm 0.16$ & $4.61 \pm 0.15$ \\
\hline Smart Explorer & $2.93 \pm 0.06$ & $2.90 \pm 0.04$ & $3.34 \pm 0.08$ & $3.93 \pm 0.10$ \\
\hline Average & 3.06 & 3.00 & 3.27 & 4.23 \\
\hline
\end{tabular}

Table 7: Average run time (in milliseconds) of the Temporal Backtracking algorithm

Table 7 shows the average run time in milliseconds of TB algorithm. From the results we can see that as the value of $k$ increases, the overall average run time increases as well. This is due to the increased amount of candidate runs. When we compare the run time of TB against DBN, we see that DBN is significantly faster than TB algorithm. Where the TB algorithm benefits over DBN is training time, because CBR system do not need training.

\subsubsection{Discussion}

In this section, we compare the TB to DBN using the benchmark. We found that the TB algorithm performed similar to the BNk2 and NNk2 for the Smart Straight Line behaviour, similar to the BN behaviour for $k<100$ and similar to the Zig-Zag behaviour for $k=100$. For the Fixed Sequence behaviour and Smart Explorer, the TB algorithm performed worse than all graphical models. From the results we can

say that for complex behaviours such as the Smart Explorer, a graphical solution is 
better. From the Zig-Zag results we can say that the TB algorithm can be affected by bias in the training set.

As noted earlier, when evaluating the TB, a few problems were noted about the specification of the algorithm. In the next section we will take a more in depth look at how the algorithm works.

\subsection{Analysis of TB}

In Section 3.1 we briefly highlighted situations where the behaviour is undefined in the algorithm. The goal of this section is to analyze which situations cause undefined behaviour in the algorithm and propose a more complete definition of the algorithm.

The main purpose of the algorithm, given a set of candidate runs, is to find a run or subset of runs similar to the problem run that result in the same action. The algorithm will eliminate non-similar runs until the remaining candidate runs reach consensus and result in the same action. The algorithm attempts to eliminate runs by comparing the current input of each run. If consensus cannot be reached, more information must be provided to the algorithm to find differences between problem and candidate runs. This results in using past input and past actions to find instances where a candidate run differs from the problem run. By increasing the actions and inputs used in the comparison, we can possibly further discriminate between equal candidate runs.

We previously defined the TB algorithm in Algorithm 1. The pseudo code gives us a general overview of how the algorithm eliminates candidate runs until consensus is reached. There are some cases that are not handled by the algorithm. The following 
sections will look at those cases.

\subsubsection{Consensus not reached at the end of the problem run}

The first case we will look at is the behaviour of the TB algorithm when it reaches the end of the candidate run and problem run, but consensus has not been reached. For the following example, inputs are continuous values, while actions are discrete values. The similarity values for actions are defined by Equation 5 and the similarity values for inputs are defined in Table 8. In addition, the PT and the ST will be arbitrarily set at 0.75 .

$$
\operatorname{sim}\left(a_{1}, a_{2}\right)= \begin{cases}1 & , a_{1}=a_{2} \\ 0 & , \text { otherwise }\end{cases}
$$

\begin{tabular}{|c|c|c|c|}
\hline & $i_{0}$ & $i_{1}$ & $i_{2}$ \\
\hline \hline$i_{0}$ & 1.0 & 0.9 & 0.9 \\
\hline$i_{1}$ & 0.9 & 1.0 & 0.8 \\
\hline$i_{2}$ & 0.9 & 0.8 & 1.0 \\
\hline
\end{tabular}

Table 8: The similarity matrix for inputs

Consider the following run:

- $r_{p}: i_{1} \stackrel{a}{\rightarrow} i_{0} \stackrel{?}{\rightarrow}$

- $r_{c_{1}}: i_{0} \stackrel{a}{\rightarrow} i_{0} \stackrel{b}{\rightarrow}$ 


$$
\text { - } r_{c_{2}}: i_{2} \stackrel{a}{\rightarrow} i_{0} \stackrel{c}{\rightarrow}
$$

In this example, we can see that the candidate runs do not have consensus. Because there is no consensus, the TB algorithm must go back into the past. When the algorithm checks the previous action, it cannot eliminate any of the candidate runs because the similarity of the actions are all above the threshold. Because no candidate runs were eliminated and there is still no consensus between the candidate runs, the TB algorithm must look back further at the input that generated the past action. When the TB algorithm checks the previous input, it cannot eliminate any of the candidate runs because the similarity of the inputs are above the threshold. At this point in the TB algorithm, we have reached the end of the candidate and problem runs and there is still no consensus between the candidate runs.

Algorithm 1 does not define the action that the TB algorithm should take for this case. While implementing the TB algorithm, it was assumed that if there was still no consensus, the candidate run with the input farthest in the past with the closest similarity to the problem run would be the run selected from the TB algorithm and all other candidate runs would be eliminated. For this example, the run that would be selected would be run $r_{c_{1}}$. The problem with using the farthest in the past input with the highest similarity is that there can be a situation where all inputs in the past have equal similarity. If at this point the algorithm cannot reach consensus, the algorithm selects a candidate run at random.

\subsubsection{Time Slice versus Run Similarity}

Consider a similar example where the TB algorithm cannot eliminate any of the candidate runs, and the input farthest in the past of both candidate runs has equal 
similarity. As with the previous example, we will keep the PT and ST the same arbitrary value(0.75).

\begin{tabular}{|c|c|c|c|}
\hline & $i_{0}$ & $i_{1}$ & $i_{2}$ \\
\hline \hline$i_{0}$ & 1.0 & 0.9 & 0.76 \\
\hline$i_{1}$ & 0.76 & 1.0 & 0.76 \\
\hline$i_{2}$ & 0.76 & 0.76 & 1.0 \\
\hline
\end{tabular}

Table 9: The similarity matrix between inputs

- $r_{p}: i_{0} \stackrel{a}{\rightarrow} i_{2} \stackrel{d}{\rightarrow} i_{1} \stackrel{a}{\rightarrow} i_{0} \stackrel{?}{\rightarrow}$

- $r_{c_{1}}: i_{0} \stackrel{a}{\rightarrow} i_{2} \stackrel{d}{\rightarrow} i_{1} \stackrel{a}{\rightarrow} i_{0} \stackrel{b}{\rightarrow}$

- $r_{c_{2}}: i_{0} \stackrel{a}{\rightarrow} i_{0} \stackrel{d}{\rightarrow} i_{0} \stackrel{a}{\rightarrow} i_{0} \stackrel{c}{\rightarrow}$

In this example, we see that all the similarities for actions and inputs are above the threshold and that there is no consensus between the candidate runs. As with the previous example, the algorithm reaches the input farthest in the past without eliminating any of the candidate runs. At this point, the TB algorithm will assume that because all the inputs farthest in the past have similarity greater than the threshold, it will randomly select a candidate run to keep and eliminate the rest. The problem with this assumption is that the TB algorithm does not take into account similarities from other parts of the run. For example, the TB algorithm could eliminate run $r_{c_{1}}$ even though the run is identical to the problem run, but because it has the same past input as the other candidate run, it is randomly chosen to be eliminated. 
The TB algorithm bases this decision on two properties. The first is that similarity of inputs and similarity of actions are independent of each other. The second property is that the algorithm cares only if the similarity is over the threshold or under the threshold and it does not care about the difference between similarity and threshold. As a result of these two properties, the similarity between a problem and a candidate run is determined by the number of consecutive similarity values of actions and inputs that are over the threshold. If runs can be viewed as sequences of symbols then the algorithm can be simply viewed as finding the candidate run with the longest suffix.

Before we define an equation for the longest common suffix, we must first define an equation for how the TB algorithm handles case similarity. The TB algorithm recursively compares runs starting from the current input and moving into the past until the similarity of an input or action is below the threshold. This means that cases are compared first by action then by input, but the input is compared only when the similarity of the action is greater than the threshold. For example, $r^{a}(1)$ will be compared if $r^{i}(0)$ is above the threshold, and $r^{i}(1)$ will be compared if $r^{a}(1)$ is above the threshold, and so on. We can define a recursive definition of measuring the longest suffix using the equation below. 


$$
L C\left(r_{p}(k), r_{c}(k)\right)= \begin{cases}2+L C\left(r_{p}(k+1), r_{c}(k+1)\right) & \text {,if } \operatorname{sim}\left(r_{p}^{a}(k), r_{c}^{a}(k)\right)>S T \wedge \\ & \operatorname{sim}\left(r_{p}^{i}(k), r_{c}^{i}(k)\right)>P T \\ 1 & , \text { if } \operatorname{sim}\left(r_{p}^{a}(k), r_{c}^{a}(k)\right)>S T \wedge \\ & \operatorname{sim}\left(r_{p}^{i}(k), r_{c}^{i}(k)\right) \leq P T \\ 0 & \text {, otherwise }\end{cases}
$$

Where $r_{j}(k)$ is the case of run $j, k$ th cases into the past. The most current case in a given run is defined as $r_{j}(0)$ and the case farthest in the past is defined as $r_{j}(n)$. $r_{j}^{a}(k)$ is defined as the action of case $r_{j}(k)$ and $r_{j}^{i}(k)$ is defined as the input of that same case. PT is defined as the problem threshold, and $S T$ is defined as the solution threshold.

The TB algorithm for comparing runs can be defined as follows:

$$
\operatorname{sim}\left(r_{p}, r_{c}\right)=L C\left(r_{p}(0), r_{c}(0)\right)
$$

where $r_{p}$ is the problem run, $r_{c}$ is a candidate run.

There are a couple of things we can notice about Equation 7 . The first is that similarities are not bound to $[0,1]$. This equation can be modified to normalize all candidate similarities by the length of the problem run. The second is that using just the longest suffix can result in ties between candidate runs that have not reached consensus and does not provide enough information to break the ties. The algorithm not having enough information to break ties could be the result of a relaxed threshold. 
There are a few solutions to fix this problem. The first solution is to use a strict threshold, but the problem with this is that it could lead to a false negative when comparing inputs and actions. The second solution is to keep track of the total similarity of the longest suffix. By doing this, ties can be broken by the higher total similarity.

\subsubsection{Length of Candidate run (Cut-off point)}

In the previous examples, we looked at how the TB algorithm analyzes candidate runs based on individual inputs or actions and how it solves candidate runs that are similar. In those examples, each of the candidate runs has a length equal to the problem run. In real-world examples, candidate runs are not always the same length as the problem run. They can be longer or shorter than the problem run. In this section, we will look at how the TB algorithm handles longer or shorter candidate runs.

First, we will look at how the TB algorithm handles candidate runs that are longer than the problem run. For the following examples, we will use the similarity action function defined in Equation 5. For inputs, we will use the equation defined in Equation 8.

$$
\operatorname{sim}\left(i_{1}, i_{2}\right)= \begin{cases}1 & , i_{1}=i_{2} \\ 0 & , \text { otherwise }\end{cases}
$$

Consider the problem run given below and two candidate runs; one is longer than the problem run and one is the same length as the problem run: 
- $r_{p}: A \stackrel{a}{\rightarrow} B \stackrel{?}{\rightarrow}$

- $r_{c_{1}}: A \stackrel{a}{\rightarrow} B \stackrel{b}{\rightarrow}$

- $r_{c_{2}}: C \stackrel{c}{\rightarrow} A \stackrel{a}{\rightarrow} B \stackrel{c}{\rightarrow}$

In this example, we can see that the second run is longer than the problem run and there is no consensus between the two candidate runs. When the TB algorithm compares the problem run to the second run, it will reach the end of the problem run, but will not reach the end of the second candidate run. The algorithm previously defined in Algorithm 1 does not specify what happens at this point, but we assume that all remaining states and the candidate run longer than the problem run length are ignored because there is nothing to compare to. Thus the similarity calculation is based on temporal distance from the most current event.

Next, we will look at how the TB algorithm handles candidate runs that are shorter than the problem run. As with the previous example, we will assume that all similarity values are above the threshold.

Consider the problem run given below and the two candidate runs; one is shorter than the problem run and the other is the same length as the problem run:

$$
\begin{aligned}
& \text { - } r_{p}: A \stackrel{a}{\rightarrow} B \stackrel{?}{\rightarrow} \\
& \text { - } r_{c_{1}}: A \stackrel{a}{\rightarrow} B \stackrel{b}{\rightarrow} \\
& \text { - } r_{c_{2}}: B \stackrel{c}{\rightarrow}
\end{aligned}
$$

In this example, we see that the second candidate run is shorter than the problem run. As with the previous example, the TB algorithm does not specify what happens 
when a candidate run is shorter. As a result, we assume that candidate runs that are shorter than the problem run are eliminated as possible candidate runs if consensus is not reached by the time the TB algorithm has reached the end of the candidate run. For example:

- $r_{p}: A \stackrel{\alpha}{\rightarrow} A \stackrel{a}{\rightarrow} B \stackrel{?}{\rightarrow}$

- $r_{c_{1}}: A \stackrel{a}{\rightarrow} B \stackrel{b}{\rightarrow}$

- $r_{c_{2}}: C \stackrel{\delta}{\rightarrow} C \stackrel{c}{\rightarrow} B \stackrel{c}{\rightarrow}$

In the above example, the second candidate run is eliminated because the most recent substitution is more recent than the length of the first candidate run.

- $r_{p}: A \stackrel{\alpha}{\rightarrow} A \stackrel{a}{\rightarrow} B \stackrel{?}{\rightarrow}$

- $r_{c_{1}}: A \stackrel{a}{\rightarrow} B \stackrel{b}{\rightarrow}$

- $r_{c 2}: C \stackrel{\delta}{\rightarrow} A \stackrel{a}{\rightarrow} B \stackrel{c}{\rightarrow}$

In this example, the TB algorithm will reach the end of the first candidate run without eliminating any of the candidate runs because they are the same as the

problem run. When the TB algorithm backtracks farther than the length of the first candidate run, it will be eliminated.

\subsubsection{Noise}

The TB algorithm is sensitive to small difference between runs when similarity calculations for input or actions use a Boolean metric. Consider the example below, which shows a problem run with two candidate runs: 
- $r_{p}: \ldots \rightarrow C \stackrel{\lambda}{\rightarrow} A \stackrel{\alpha}{\rightarrow} B \stackrel{?}{\rightarrow}$

- $r_{c_{1}}: F \stackrel{\mu}{\rightarrow} D \stackrel{\alpha}{\rightarrow} B \stackrel{\beta}{\rightarrow}$

- $r_{c_{2}}: C \stackrel{\lambda}{\rightarrow} A \stackrel{\theta}{\rightarrow} B \stackrel{\sigma}{\rightarrow}$

When the algorithm starts looking back, it will eliminate the second candidate run because $r_{c_{2}}^{a}(1)$ is different from $r_{p}^{a}(1)$. Even though the second candidate run is more similar to the problem run than the first run, it eliminates the second run because one of the more recent actions is different from the problem run.

\subsection{Discussion}

In this chapter, we looked at two different solutions for LFO; a graphical model solution and a CBR solution. When we compared both solutions together we found that CBR performed worse than the graphical model solution. The CBR performed significantly worse than the graphical model solution when learning the Smart Explorer behaviour. We can conclude that for the agents in the benchmark, the graphical model solution is better.

In addition we have analyzed the TB algorithm. In our analysis of the algorithm, we can simplify its behaviour to a similarity function that will be based on the longest suffix that is similar to the problem run. When analyzing this behaviour, we see that the algorithm is sensitive to the threshold value, bias in the candidate runs, varying candidate run lengths, and random noise when using a Boolean metric. The main factors of the algorithm's sensitivity are mainly caused by comparisons of actions and inputs based on temporal offset, and by basing comparisons on whether the 
similarity is above a given threshold. As a result, these factors propose an overly specific solution for LFO.

In the next section, we will look at other similarity functions that can be used as an improvement to the TB algorithm and we will generalize a framework for handling the different similarity functions. 


\section{Chapter 5}

\section{Methodology and Results of Generalizing the Case-based Reasoning Approach to Case Similarity}

In the previous section, we looked at the TB algorithm and some of the issues that can arise while using it. In this section, we will try to generalize a solution to those issues. We will try to rephrase the TB algorithm using the new framework as well as look at different similarity metrics.

\subsection{Defining the Framework}

Earlier we defined a run as a sequential list of cases; we defined a case as a 3-tuple consisting of an input, action, and past case, and we defined a run as being composed of cases. The concept of a run was described as a separate entity from a case except it has a composite relationship. For the new framework, we can introduce a new relationship between a run and a case, and this relationship is shown in Figure 10. 
We can use a Composite design pattern [15] to represent the relationship between a case and a run. In this design, we maintain that a run is composed of cases. A case is composed of a pair consisting of an input and action. As a result of the inheritance relationship, a run is also composed of an input and an action. In addition to an action and an input, a run will also contain a list of past cases.

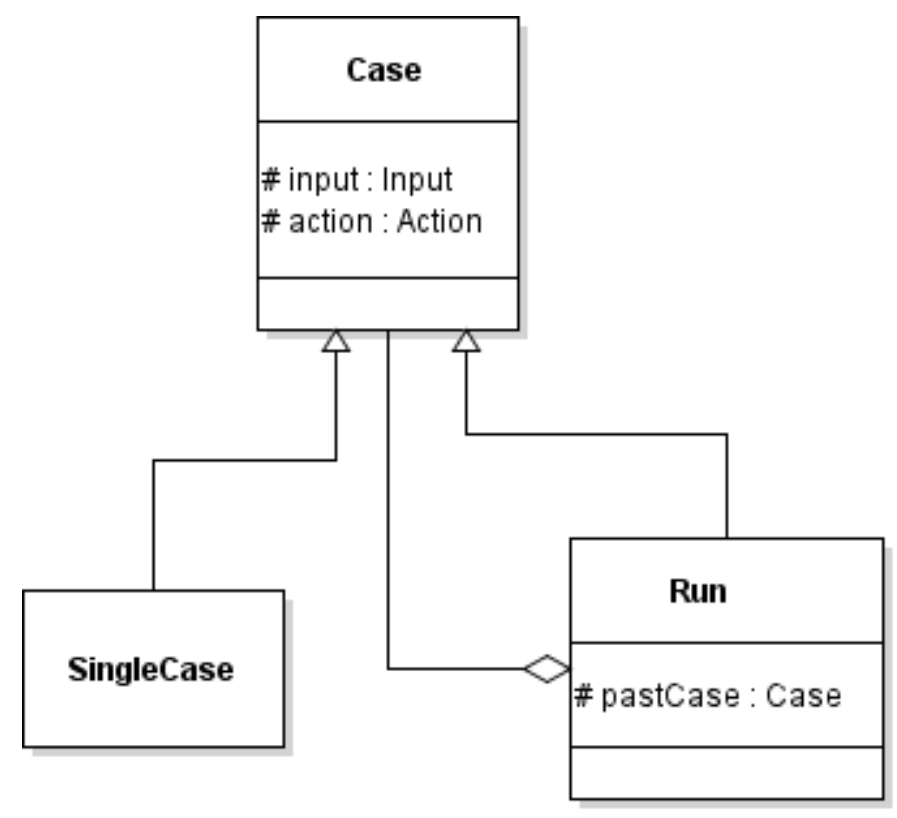

Figure 10: A class diagram showing the relationships between cases and runs

A run can now be composed of smaller runs. This composite model can help to organize more complicated situations. Consider the Smart Explorer behaviour from the benchmark test. The goal of the Smart Explorer behaviour is to visit every location in the map. It achieves this through the sub-goals of moving toward a dirt object if found, and wandering randomly. Some of the sub-goals consist of multiple steps and can be defined using the new case run relationship. Each of the sub-goal becomes its own run, composed of cases that denote the steps needed to complete 


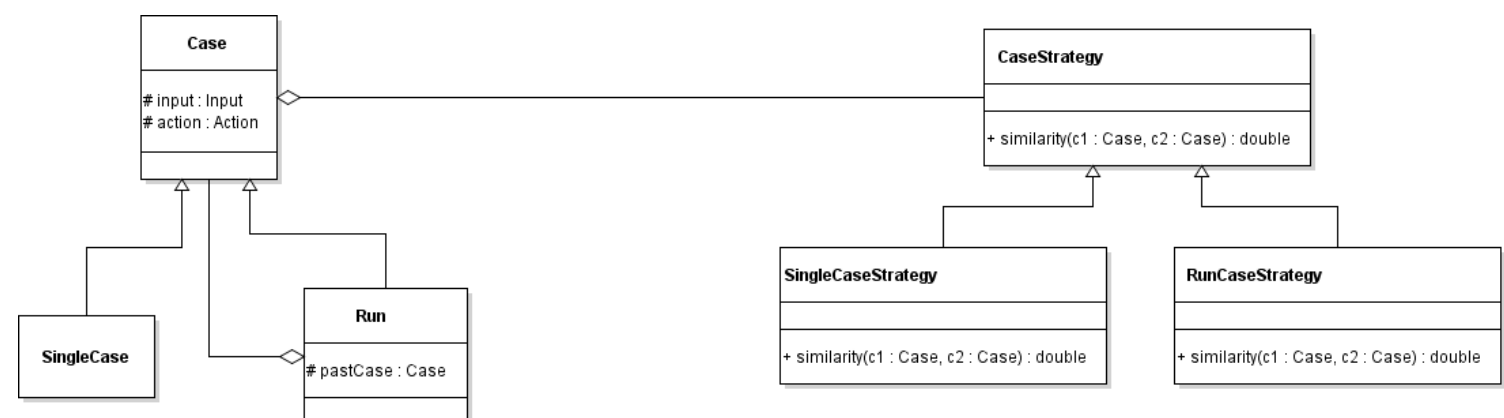

Figure 11: A class diagram showing the relationship between cases and similarity strategies used for case comparison

the sub-goal.

In CBR, cases are compared using a similarity function. With the introduction of the inheritance relationship between runs and cases, runs can also be compared using a similarity function. Case similarity functions and run similarity functions are different. With cases, inputs are compared with inputs and outputs are compared with outputs. With a run, an input in one run can be compare with an input of any case in another run. To allow separate similarity functions for cases and runs while maintaining the inheritance relationship, we must introduce the Strategy design pattern [15] and the resulting relationship is defined in Figure 11. Using this pattern decouples the similarity strategy from the cases and allows each case or run to implement its own similarity function.

In the following sections, we will take a look at some run similarity strategies that can be used, and compare their performance with that of the TB algorithm. 


\subsection{Run Similarity}

The first solution we will look at is an implementation of the RunCaseStrategy that will calculate the similarity of the entire run. The similarity calculation that we will be using will be an average. This is described in Equation 9 .

Before we explain the solution further, we must first introduce three helper functions. The first function, denoted by Action $(c)$ will return the action of case $c$. The second helper function, Input(c), will return the input of case $c$. The last helper function, $\operatorname{sim}(x, y)$, will return the similarity between $x$ and $y$. To find the similarity of two runs we must take into account the similarities of all actions and inputs in the run. We define a new function $\operatorname{runSim}(P, C)$, described in Equation 9, to find the similarity between the problem run, $P$, and a candidate run, $C$.

$$
\begin{aligned}
\operatorname{runSim}(P, C)= & \frac{1}{2|P|+1}\left[\operatorname{sim}\left(\operatorname{State}\left(P_{0}\right), \operatorname{State}\left(C_{0}\right)\right)+\right. \\
& \sum_{i=1}^{|P|}\left[\operatorname{sim}\left(\operatorname{State}\left(P_{i}\right), \operatorname{State}\left(C_{i}\right)\right)+\right. \\
& \left.\left.\operatorname{sim}\left(\operatorname{Action}\left(P_{i}\right), \operatorname{Action}\left(C_{i}\right)\right)\right]\right]
\end{aligned}
$$

The function $\operatorname{run} \operatorname{Sim}(P, C)$ calculates the average similarity of the candidate run, $C$, with the problem run, $P$. In the function we are comparing the action and inputs based on the temporal distance from the current input and we are interested in finding a candidate run similar to the problem run. As a results we will normalize the similarity based on the size of the problem run. By restricting comparisons to the size of the problem run, cases farther in the past than the size of the problem run are not included in the similarity calculation. One thing to note about this function, 
each input and action has equal weighting toward the run similarity average. As we can see with the TB algorithm, having equal weights can lead to some unexpected behaviour.

Consider the Zig-Zag behaviour from the benchmark test. The agent's next turn direction is dependent on the previous turn direction. If we look at all the action before the previous turn direction, we see that they have no relevance on the next turn direction. Thus, the runSim function should represent the fact that older information has lesser impact on future events, and the past inputs and states should be weighted accordingly. This leads us to the new Equation 10:

$$
\begin{aligned}
\operatorname{runSim}(P, C)= & \frac{1}{2|P|+1}\left[\operatorname{sim}\left(\operatorname{State}\left(P_{0}\right), \operatorname{State}\left(C_{0}\right)\right)+\right. \\
& \sum_{i=1}^{|P|}\left[\operatorname{sim}\left(\operatorname{State}\left(P_{i}\right), \operatorname{State}\left(C_{i}\right)\right) * w_{i}(\theta)+\right. \\
& \left.\left.\operatorname{sim}\left(\operatorname{Action}\left(P_{i}\right), \operatorname{Action}\left(C_{i}\right)\right) * w_{i}(\theta)\right]\right]
\end{aligned}
$$

In this new equation, we have added the term $w_{t}(\theta)$ as the weight at time $t$ with configuration $\theta$. By adding weights to the equation, we assume that as you go farther back in time, the weight of past actions or past inputs is reduced. In the following sections, we will look at some simple weights applied to the run similarity equation. We will look at three different decay weights: constant decay, exponential decay and a Gaussian decay. For the following weights we will compare the results with the TB at $k=10$. In the previous section it was identified that $k=10$ was the optimum for accuracy for some of the behaviours, except for the Smart Explorer behaviour. 
In addition, we will use the same similarity calculation that was used for the TB algorithm.

For the following experiments, we will only using a $k=10$ and comparing to the TB algorithm at $k=10$. This restriction is due to the long run times of the edit distance and Jaccard distance taking each a week to run at $k=10$.

\subsubsection{Comparison With TB}

The run similarity algorithm takes into account the whole run for similarity calculations, while the TB algorithm handles calculation of actions and states independently. There are some advantages to using the run similarity algorithm over the TB algorithm.

The TB algorithm is sensitive to noise when similarity calculations for state or actions uses a Boolean metric. Consider the example below, which shows a problem run with two candidate runs:

- $r_{p}: \ldots \rightarrow C \stackrel{\lambda}{\rightarrow} A \stackrel{\alpha}{\rightarrow} B \stackrel{?}{\rightarrow}$

- $r_{c_{1}}: F \stackrel{\mu}{\rightarrow} D \stackrel{\alpha}{\rightarrow} B \stackrel{\beta}{\rightarrow}$

- $r_{c_{2}}: C \stackrel{\lambda}{\rightarrow} A \stackrel{\theta}{\rightarrow} B \stackrel{\sigma}{\rightarrow}$

The candidate cases do not have consensus. Thus, the TB algorithm needs to look back. It eliminates the second run because one of the more recent actions is different from the problem run.

Now let us look at the same example, but use the run similarity function. In this example, we will use a constant decay weight function, where $C$ is 0.1 , and we will 
use a Boolean metric for similarity calculations. The breakdown of the similarities of each candidate run is shown below.

\begin{tabular}{|c|c|c|c|c|c|c|}
\hline Problem Run & $\mathrm{C}$ & $\lambda$ & $\mathrm{A}$ & $\alpha$ & $\mathrm{B}$ & Average Similarity \\
\hline Weight & 0.6 & 0.7 & 0.8 & 0.9 & 1 & \\
\hline \hline Candidate Run 1 & $\mathrm{F}$ & $\mu$ & $\mathrm{D}$ & $\alpha$ & $\mathrm{B}$ & \\
\hline Similarity Run 1 & 0 & 0 & 0 & 0.9 & 1 & 0.38 \\
\hline Candidate Run 2 & $\mathrm{C}$ & $\lambda$ & $\mathrm{A}$ & $\theta$ & $\mathrm{B}$ & \\
\hline Similarity Run 2 & 0.6 & 0.7 & 0.8 & 0 & 1 & 0.62 \\
\hline
\end{tabular}

Table 10: Breakdown over a run of similarity calculations for temporal backtracking algorithm and run similarity with constant decay weight function where $C=$ 0.1

As we can see, the similarity of run 2 is higher than run 1 and the run similarity algorithm will select action $\sigma$ from candidate run 2. In this example, we can see that the run similarity algorithm is not affected by noise.

Now let us look at an example where the TB algorithm selects a more likely similar run than the run similarity algorithm does. As with the first example, we will use a constant decay weight function where $\mathrm{C}$ is 0.1 , but instead of using a Boolean metric, we will use a continuous similarity metric. We will also set the threshold for the TB algorithm to 0.5. Consider the following problem and candidate runs, and their candidate run values:

$$
\begin{aligned}
& \text { - } r_{p}: \ldots D^{\delta} \rightarrow C \stackrel{\lambda}{\rightarrow} A \stackrel{\alpha}{\rightarrow} B \stackrel{?}{\rightarrow} \\
& \text { - } r_{c_{1}}: F \stackrel{\varepsilon}{\rightarrow} E \stackrel{\mu}{\rightarrow} D \stackrel{\theta}{\rightarrow} B \stackrel{\beta}{\rightarrow}
\end{aligned}
$$


- $r_{c_{2}}: C \stackrel{\rho}{\rightarrow} M \stackrel{\lambda}{\rightarrow} A \stackrel{\alpha}{\rightarrow} B \stackrel{\sigma}{\rightarrow}$

\begin{tabular}{|c|c|c|c|c|c|c|c|c|}
\hline Problem Run & $\mathrm{D}$ & $\delta$ & $\mathrm{C}$ & $\lambda$ & $\mathrm{A}$ & $\alpha$ & $\mathrm{B}$ & Average Sim \\
\hline Weight & 0.4 & 0.5 & 0.6 & 0.7 & 0.8 & 0.9 & 1 & \\
\hline \hline Candidate Run 1 & $\mathrm{D}$ & $\varepsilon$ & $\mathrm{E}$ & $\mu$ & $\mathrm{D}$ & $\theta$ & $\mathrm{B}$ & \\
\hline Vanilla Sim Run 1 & 1 & 0.75 & 0.5 & 0.5 & 0.5 & 0.5 & 1 & 0.679 \\
\hline Weighted Sim Run 1 & 0.4 & 0.375 & 0.3 & 0.35 & 0.4 & 0.45 & 1 & 0.468 \\
\hline Candidate Run 2 & $\mathrm{N}$ & $\rho$ & $\mathrm{M}$ & $\lambda$ & $\mathrm{A}$ & $\alpha$ & $\mathrm{B}$ & \\
\hline Vanilla Sim Run 2 & 0 & 0 & 0 & 1 & 1 & 1 & 1 & 0.571 \\
\hline Weighted Sim Run 2 & 0 & 0 & 0 & 0.7 & 0.8 & 0.9 & 1 & 0.486 \\
\hline
\end{tabular}

Table 11: Breakdown over a run of similarity calculations for temporal backtracking algorithm and run similarity with constant decay weight function where $C=$ 0.1

In this problem, candidate run 1 has a higher vanilla average similarity than run 2. We define the vanilla similarity run as the run before weights are applied. When weights are applied to the vanilla similarity we can see that candidate run 2 has a higher average similarity. If the TB algorithm is used to decide on the candidate run, it will select run 1 , because halfway through run 2 the similarity is below the threshold and thus is eliminated. If the run similarity algorithm is used, run 2 will be selected because it has the higher average similarity. In candidate run 2 , the early half of the run has no similarity to the problem run, yet when using the run similarity algorithm is used, candidate run 2 is selected. This selection is due to the weighting of the similarities, and using general weights could lead to situations in which the 
less similar run may be selected.

Now let us look at an example where there are different run lengths. In this example, we will use a constant decay weight function, where $C$ is 0.1 , and we will use a Boolean metric for similarity calculations. Consider the following problem and candidate runs:

- $r_{p}: \ldots C \stackrel{\lambda}{\rightarrow} A \stackrel{\alpha}{\rightarrow} B \stackrel{?}{\rightarrow}$

- $r_{c_{1}}: D \stackrel{\alpha}{\rightarrow} B \stackrel{\beta}{\rightarrow}$

- $r_{c_{2}}: C \stackrel{\lambda}{\rightarrow} A \stackrel{\theta}{\rightarrow} B \stackrel{\sigma}{\rightarrow}$

In this problem, candidate run 1 is shorter than run 2. When TB algorithm is applied to this problem, it will eliminate run 2 because the previous action is different from the problem run. Although candidate run 2 is more similar than run 1, having the similarity of an action or input lower than the threshold closer to $t=0$, results in earlier elimination with the TB algorithm. When the run similarity algorithm is applied to the same problem, the outcome is different. 


\begin{tabular}{|c|c|c|c|c|c|c|}
\hline Problem Run & $\mathrm{C}$ & $\lambda$ & $\mathrm{A}$ & $\alpha$ & $\mathrm{B}$ & Average Similarity \\
\hline Weight & 0.6 & 0.7 & 0.8 & 0.9 & 1 & \\
\hline \hline Candidate Run 1 & & & $\mathrm{D}$ & $\alpha$ & $\mathrm{B}$ & \\
\hline Similarity Run 1 & 0 & 0 & 0 & 0.9 & 1 & 0.38 \\
\hline Candidate Run 2 & $\mathrm{C}$ & $\lambda$ & $\mathrm{A}$ & $\theta$ & $\mathrm{B}$ & \\
\hline Similarity Run 2 & 0.6 & 0.7 & 0.8 & 0 & 1 & 0.62 \\
\hline
\end{tabular}

Table 12: Breakdown over a run of similarity calculations for temporal backtracking algorithm and run similarity with constant decay weight function where $C=$ 0.1

When the weights are applied to each of the candidate runs, we see that the average similarity of run 2 is higher than that of run 1 . We see that the run selected from the run similarity algorithm is different from that selected by the TB algorithm. The run similarity takes into account all corresponding actions and inputs in the candidate runs. By doing this, noise that appears close to the current state has less of an effect in deciding which candidate run is closer.

In the current benchmark, noise appears only in the actions. The environment used in the benchmark is static and thus no noise is generated from the environment. As a result, the results using the benchmark does not fully display the benefit of calculating the similarity based on the whole run instead of individual actions and inputs. Future work should include enhancing the benchmark with the possibility of using noisy inputs, so you can test whether your approach can be better. 


\subsubsection{Constant Decay}

The first decay weight we will look at is the constant decay weight. A constant decay weight function is described by Equation 11. In this equation, $C$ is the decay constant and the value of the weight function decreases at a constant rate over time. The weight function was compared against the TB algorithm at $k=10$. Four different values of $C$ were used: $0.5,0.2,0.1$, and 0.05 . These values were chosen arbitrarily.

$$
w_{t}(C)=1-C * t
$$




\begin{tabular}{|c|c|c|c|}
\hline Rate of deterioration & 0.5 & 0.2 & $\mathrm{~TB} \mathrm{k}=10$ \\
\hline \hline Smart Random & $30.80 \pm 1.08$ & $30.62 \pm 1.37$ & $30.63 \pm 0.35$ \\
\hline Smart Straight Line & $76.67 \pm 1.87$ & $71.26 \pm 2.35$ & $93.28 \pm 0.30$ \\
\hline Zig-Zag & $58.53 \pm 0.54$ & $55.07 \pm 0.93$ & $44.53 \pm 1.76$ \\
\hline Fixed Sequence & $25.14 \pm 0.99$ & $24.94 \pm 1.24$ & $25.44 \pm 1.22$ \\
\hline Smart Explorer & $8.39 \pm 7.04$ & $10.78 \pm 14.53$ & $5.50 \pm 6.60$ \\
\hline Average & 39.91 & 38.53 & 39.88 \\
\hline
\end{tabular}

\begin{tabular}{|c|c|c|c|}
\hline Rate of deterioration & 0.1 & 0.05 & $\mathrm{~TB} \mathrm{k}=10$ \\
\hline \hline Smart Random & $30.68 \pm 1.59$ & $30.48 \pm 1.05$ & $30.63 \pm 0.35$ \\
\hline Smart Straight Line & $65.82 \pm 1.95$ & $59.53 \pm 1.28$ & $93.28 \pm 0.30$ \\
\hline Zig-Zag & $52.91 \pm 0.45$ & $51.09 \pm 0.45$ & $44.53 \pm 1.76$ \\
\hline Fixed Sequence & $25.05 \pm 0.85$ & $24.28 \pm 1.46$ & $25.44 \pm 1.22$ \\
\hline Smart Explorer & $11.84 \pm 13.98$ & $10.62 \pm 12.16$ & $5.50 \pm 6.60$ \\
\hline Average & 37.26 & 35.2 & 39.88 \\
\hline
\end{tabular}

Table 13: Run Similarity Accuracy of Time Dependent $k=10$ with constant decay 


\begin{tabular}{|c|c|c|c|}
\hline Rate of deterioration & 0.5 & 0.2 & TB F1 $k=10$ \\
\hline \hline Smart Random & $30.77 \pm 1.08$ & $30.55 \pm 1.36$ & $30.62 \pm 0.36$ \\
\hline Straight Line & $76.57 \pm 1.95$ & $71.22 \pm 2.38$ & $50.66 \pm 1.46$ \\
\hline Zig-Zag & $39.39 \pm 1.28$ & $39.98 \pm 1.09$ & $25.44 \pm 0.51$ \\
\hline Fixed Sequence & $24.73 \pm 0.82$ & $24.19 \pm 1.29$ & $24.56 \pm 1.35$ \\
\hline Smart Explorer & $11.21 \pm 5.58$ & $11.94 \pm 7.20$ & $8.14 \pm 4.91$ \\
\hline Average & 36.53 & 35.58 & 27.88 \\
\hline
\end{tabular}

\begin{tabular}{|c|c|c|c|}
\hline Rate of deterioration & 0.1 & 0.05 & TB F1 $k=10$ \\
\hline \hline Smart Random & $30.64 \pm 1.58$ & $30.42 \pm 0.99$ & $30.62 \pm 0.36$ \\
\hline Straight Line & $65.81 \pm 2.00$ & $59.51 \pm 1.30$ & $50.66 \pm 1.46$ \\
\hline Zig-Zag & $39.84 \pm 0.82$ & $39.71 \pm 1.41$ & $25.44 \pm 0.51$ \\
\hline Fixed Sequence & $24.51 \pm 0.80$ & $23.68 \pm 1.44$ & $24.56 \pm 1.35$ \\
\hline Smart Explorer & $11.58 \pm 7.62$ & $12.23 \pm 7.76$ & $8.14 \pm 4.91$ \\
\hline Average & 34.48 & 33.11 & 27.88 \\
\hline
\end{tabular}

Table 14: Run Similarity F1-measure of Time Dependent $k=10$ with constant decay

From the results, and comparing with Table 6, we see that for the Fixed Sequence behaviour and the Smart Random behaviour the constant decay weight performed about the same as the TB algorithm. The constant decay weights performed as expected when trying to imitate the Smart Random behaviour. The Smart Random behaviour has of large percentage of its behaviour that is random in nature with some 
percentage of the behaviour not random (moving toward a dirt object). As a result, there should be similar results between the run similarity and the TB algorithm.

The Smart Straight Line behaviour, performs worse than the TB algorithm for each of the four $C$ values. We can see that as the constant value gets smaller, it worsens the performance of the weight function. One possible explanation for this is the effect of including less important candidates into the similarity calculation to bias one action over another. One problem with using a general weight function is that the weight function is independent of the values in a candidate run. This means that actions or inputs of all candidate runs, regardless of value, at time $t$ will have the same weights applied to it. As the value of $C$ decreases, the weighting of the similarity decreases slower. As a result, the similarity of actions or inputs farther in the past can influence the similarity by either inflating the similarity or deflating the similarity of the run.

For the Zig-Zag behaviour we can see that higher rates of deterioration result in a better performance from the TB algorithm. For all of the deterioration rate, they have a higher accuracy than the TB algorithm. By taking into account the similarity of past cases, the run similarity metric can select the more similar run.

For the Smart Explorer behaviour we see that although the accuracy for each of the different values of $C$ is higher than the TB algorithm, the margin of error is still very large. This margin of error and low accuracy is due to the STAND action. The agent takes only this action when it cannot move to an adjacent location because it has already visited that location. One possible explanation is that the internal state of the Smart Explorer behaviour is too complicated to be broken down into $k$ candidate runs. The order in which the Smart Explorer visits each location does not 
matter. This means that the number of possible solutions to competing the map is huge. Each full run from the start of the run to the STAND action represents one possible solution. The likelihood of finding the correct solution to the problem by looking at only a small subset of the solution space is very small.

\begin{tabular}{|c|c|c|c|}
\hline Rate of deterioration & 0.5 & 0.2 & TB k =10 \\
\hline \hline Smart Random & $6.67 \pm 0.55$ & $6.99 \pm 0.42$ & $3.02 \pm 0.07$ \\
\hline Smart Straight Line & $6.89 \pm 0.29$ & $6.71 \pm 0.29$ & $3.16 \pm 0.13$ \\
\hline Zig-Zag & $6.33 \pm 0.55$ & $6.79 \pm 0.54$ & $2.96 \pm 0.02$ \\
\hline Fixed Sequence & $4.75 \pm 2.15$ & $4.71 \pm 1.98$ & $2.94 \pm 0.08$ \\
\hline Smart Explorer & $6.26 \pm 0.43$ & $6.26 \pm 0.63$ & $2.90 \pm 0.04$ \\
\hline Average & 6.18 & 6.29 & 3.00 \\
\hline
\end{tabular}

\begin{tabular}{|c|c|c|c|}
\hline Rate of deterioration & 0.1 & 0.05 & TB k $=10$ \\
\hline \hline Smart Random & $7.31 \pm 0.68$ & $7.14 \pm 0.32$ & $3.02 \pm 0.07$ \\
\hline Smart Straight Line & $6.87 \pm 0.37$ & $6.84 \pm 0.39$ & $3.16 \pm 0.13$ \\
\hline Zig-Zag & $6.73 \pm 0.46$ & $6.84 \pm 0.67$ & $2.96 \pm 0.02$ \\
\hline Fixed Sequence & $7.29 \pm 1.24$ & $7.97 \pm 1.63$ & $2.94 \pm 0.08$ \\
\hline Smart Explorer & $6.33 \pm 0.42$ & $6.13 \pm 0.36$ & $2.90 \pm 0.04$ \\
\hline Average & 6.91 & 6.98 & 3.00 \\
\hline
\end{tabular}

Table 15: Average run time (in milliseconds) of Temporal Backtracking with run similarity using a constant weight function

From the results in Table 15 we can see that as the rate of deterioration decreases, 
the overall average run time increases. This increase is most notable with the Fixed Sequence agent. The increase in run time of the Fixed Sequence agent is most likely not caused from the run similarity metric. Increasing or decreasing the deterioration rate only changes the value of $C$ and should not change the number of cases being compared. Therefore the cause to the increase is most likely due to another part of the code or due to a service on the machine taking up CPU resources and increasing the run time. When we compare the run time of run similarity to the original TB, we see that the run time of the original TB is less. This is due to the run similarity looking through the whole problem run instead of only looking at cases until it find the first non-similar case.

\subsubsection{Exponential Decay}

The next rate we will look at is an exponential decay weight function. The exponential decay weight function is described by Equation 12. The weight function was compared against the TB algorithm at $k=10$. Four different values of $\lambda$ were used: $10,1,0.1$, and 0.01 . These values were chosen arbitrarily.

$$
w_{t}(\lambda)=e^{-\lambda t}
$$




\begin{tabular}{|c|c|c|c|}
\hline Rate of deterioration & 10 & 1 & TB $k=10$ \\
\hline \hline Smart Random & $30.78 \pm 1.67$ & $30.47 \pm 1.12$ & $30.63 \pm 0.35$ \\
\hline Smart Straight Line & $77.26 \pm 3.10$ & $76.29 \pm 2.57$ & $93.28 \pm 0.30$ \\
\hline Zig-Zag & $50.12 \pm 0.65$ & $55.35 \pm 0.89$ & $44.53 \pm 1.76$ \\
\hline Fixed Sequence & $25.19 \pm 1.07$ & $25.42 \pm 0.51$ & $25.44 \pm 1.22$ \\
\hline Smart Explorer & $13.31 \pm 15.44$ & $18.62 \pm 17.30$ & $5.50 \pm 6.60$ \\
\hline Average & 39.33 & 41.23 & 39.88 \\
\hline
\end{tabular}

\begin{tabular}{|c|c|c|c|}
\hline Rate of deterioration & 0.1 & 0.01 & TB $k=10$ \\
\hline \hline Smart Random & $30.67 \pm 1.37$ & $29.82 \pm 0.91$ & $30.63 \pm 0.35$ \\
\hline Smart Straight Line & $59.89 \pm 1.52$ & $50.58 \pm 2.31$ & $93.28 \pm 0.30$ \\
\hline Zig-Zag & $50.70 \pm 0.75$ & $49.44 \pm 0.57$ & $44.53 \pm 1.76$ \\
\hline Fixed Sequence & $24.86 \pm 1.03$ & $29.37 \pm 2.09$ & $25.44 \pm 1.22$ \\
\hline Smart Explorer & $8.33 \pm 6.47$ & $13.15 \pm 13.36$ & $5.50 \pm 6.60$ \\
\hline Average & 34.89 & 34.47 & 39.88 \\
\hline
\end{tabular}

Table 16: Run Similarity Accuracy of Exponential Decay $k=10$ compared against the temporal backtracking algorithm (TB) 


\begin{tabular}{|c|c|c|c|}
\hline Rate of deterioration & 10 & 1 & TB F1 $k=10$ \\
\hline \hline Smart Random & $30.67 \pm 1.54$ & $30.39 \pm 1.05$ & $30.62 \pm 0.36$ \\
\hline Straight Line & $77.15 \pm 3.28$ & $76.28 \pm 2.61$ & $50.66 \pm 1.46$ \\
\hline Zig-Zag & $39.58 \pm 0.84$ & $40.38 \pm 0.89$ & $25.44 \pm 0.51$ \\
\hline Fixed Sequence & $24.80 \pm 1.25$ & $24.82 \pm 0.47$ & $24.56 \pm 1.35$ \\
\hline Smart Explorer & $12.61 \pm 8.52$ & $16.29 \pm 9.36$ & $8.14 \pm 4.91$ \\
\hline Average & 36.96 & 37.63 & 27.88 \\
\hline
\end{tabular}

\begin{tabular}{|c|c|c|c|}
\hline Rate of deterioration & 0.1 & 0.01 & TB F1 $k=10$ \\
\hline \hline Smart Random & $30.62 \pm 1.36$ & $29.76 \pm 0.88$ & $30.62 \pm 0.36$ \\
\hline Straight Line & $59.83 \pm 1.52$ & $50.54 \pm 2.31$ & $50.66 \pm 1.46$ \\
\hline Zig-Zag & $39.58 \pm 0.83$ & $38.17 \pm 0.42$ & $25.44 \pm 0.51$ \\
\hline Fixed Sequence & $24.38 \pm 0.96$ & $28.67 \pm 2.37$ & $24.56 \pm 1.35$ \\
\hline Smart Explorer & $10.92 \pm 4.55$ & $14.34 \pm 8.00$ & $8.14 \pm 4.91$ \\
\hline Average & 33.07 & 32.30 & 27.88 \\
\hline
\end{tabular}

Table 17: Run Similarity F1-measure of Exponential Dependent k=10 with constant decay

From the results, we can see that the weight function performed about the same as the constant decay weight function. Although the average accuracy for Smart Explorer behaviour is higher than for the TB algorithm, the high variance makes the difference less significant. 


\begin{tabular}{|c|c|c|c|}
\hline Rate of deterioration & 10 & 1 & $\mathrm{~TB} \mathrm{k}=10$ \\
\hline \hline Smart Random & $8.13 \pm 0.66$ & $7.69 \pm 1.26$ & $3.02 \pm 0.07$ \\
\hline Smart Straight Line & $7.75 \pm 0.38$ & $7.74 \pm 0.47$ & $3.16 \pm 0.13$ \\
\hline Zig-Zag & $7.22 \pm 0.50$ & $7.61 \pm 0.70$ & $2.96 \pm 0.02$ \\
\hline Fixed Sequence & $4.99 \pm 2.27$ & $5.58 \pm 2.04$ & $2.94 \pm 0.08$ \\
\hline Smart Explorer & $7.14 \pm 0.42$ & $7.25 \pm 0.47$ & $2.90 \pm 0.04$ \\
\hline Average & 7.05 & 7.17 & 3.00 \\
\hline
\end{tabular}

\begin{tabular}{|c|c|c|c|}
\hline Rate of deterioration & 0.1 & 0.01 & TB k $=10$ \\
\hline \hline Smart Random & $8.03 \pm 0.33$ & $7.38 \pm 0.48$ & $3.02 \pm 0.07$ \\
\hline Smart Straight Line & $7.73 \pm 0.36$ & $7.44 \pm 0.27$ & $3.16 \pm 0.13$ \\
\hline Zig-Zag & $7.75 \pm 0.61$ & $7.75 \pm 0.47$ & $2.96 \pm 0.02$ \\
\hline Fixed Sequence & $8.90 \pm 1.10$ & $8.47 \pm 1.21$ & $2.94 \pm 0.08$ \\
\hline Smart Explorer & $7.06 \pm 0.59$ & $6.73 \pm 0.56$ & $2.90 \pm 0.04$ \\
\hline Average & 7.89 & 7.55 & 3.00 \\
\hline
\end{tabular}

Table 18: Average run time (in milliseconds) of Temporal Backtracking with run similarity using an exponential weight function

If we look at the result in Table 18 we can that the run similarity TB has a longer run time than the original TB. 


\subsubsection{Gaussian Decay}

Lastly, we will look at a Gaussian decay rate, described by Equation 13. As with the previous weight function we will use a $k=10$. Most of the parameters (similarity functions, training set, testing set) were left unchanged from the previous weight function. Multiple means and standard deviations were used and the results of the experiment are shown below.

$$
w(t, \mu, \sigma)=\frac{1}{\sigma \sqrt{2 \pi}} e^{-\frac{(t-\mu)^{2}}{2 \sigma^{2}}}
$$




\begin{tabular}{|c|c|c|c|c|}
\hline Mean, Std $=0.15$ & 0 & 1 & 2 & TB $k=10$ \\
\hline \hline Smart Random & $30.42 \pm 1.61$ & $30.92 \pm 1.38$ & $30.20 \pm 0.62$ & $30.63 \pm 0.35$ \\
\hline Smart Straight Line & $77.05 \pm 2.65$ & $77.03 \pm 1.39$ & $61.32 \pm 2.79$ & $93.28 \pm 0.30$ \\
\hline Zig-Zag & $50.88 \pm 0.92$ & $67.87 \pm 0.55$ & $48.12 \pm 0.71$ & $44.53 \pm 1.76$ \\
\hline Fixed Sequence & $25.34 \pm 0.71$ & $25.32 \pm 0.87$ & $25.54 \pm 1.13$ & $25.44 \pm 1.22$ \\
\hline Smart Explorer & $15.23 \pm 14.18$ & $7.41 \pm 6.46$ & $17.96 \pm 19.88$ & $5.50 \pm 6.60$ \\
\hline Average & 39.78 & 41.58 & 36.63 & 39.88 \\
\hline
\end{tabular}

\begin{tabular}{|c|c|c|c|}
\hline Mean, Std $=0.15$ & 5 & 10 & TB $k=10$ \\
\hline \hline Smart Random & $30.53 \pm 1.14$ & $31.95 \pm 1.89$ & $30.63 \pm 0.35$ \\
\hline Smart Straight Line & $48.23 \pm 1.29$ & $46.11 \pm 1.36$ & $93.28 \pm 0.30$ \\
\hline Zig-Zag & $47.42 \pm 0.88$ & $47.68 \pm 0.50$ & $44.53 \pm 1.76$ \\
\hline Fixed Sequence & $24.79 \pm 0.50$ & $25.32 \pm 0.57$ & $25.44 \pm 1.22$ \\
\hline Smart Explorer & $16.31 \pm 11.45$ & $13.64 \pm 10.20$ & $5.50 \pm 6.60$ \\
\hline Average & 33.46 & 32.94 & 39.88 \\
\hline
\end{tabular}

Table 19: Run Similarity Accuracy of Gaussian Decay $k=10$ compared against the temporal backtracking algorithm (TB) 


\begin{tabular}{|c|c|c|c|c|}
\hline Mean, Std $=0.15$ & 0 & 1 & 2 & TB F1 $k=10$ \\
\hline \hline Smart Random & $31.10 \pm 2.20$ & $30.66 \pm 0.83$ & $30.04 \pm 1.69$ & $30.62 \pm 0.36$ \\
\hline Straight Line & $45.29 \pm 1.34$ & $76.22 \pm 2.98$ & $75.81 \pm 2.16$ & $50.66 \pm 1.46$ \\
\hline Zig-Zag & $37.86 \pm 1.44$ & $39.66 \pm 1.13$ & $40.26 \pm 1.18$ & $25.44 \pm 0.51$ \\
\hline Fixed Sequence & $24.87 \pm 0.54$ & $24.66 \pm 0.84$ & $24.87 \pm 0.85$ & $24.56 \pm 1.35$ \\
\hline Smart Explorer & $22.24 \pm 5.83$ & $15.91 \pm 10.37$ & $10.28 \pm 4.30$ & $8.14 \pm 4.91$ \\
\hline Average & 32.27 & 37.42 & 36.25 & 27.88 \\
\hline
\end{tabular}

\begin{tabular}{|c|c|c|c|}
\hline Mean, Std $=0.15$ & 5 & 10 & TB F1 $k=10$ \\
\hline \hline Smart Random & $30.49 \pm 1.24$ & $30.68 \pm 1.32$ & $30.62 \pm 0.36$ \\
\hline Straight Line & $71.39 \pm 2.02$ & $69.22 \pm 2.54$ & $50.66 \pm 1.46$ \\
\hline Zig-Zag & $40.24 \pm 0.77$ & $41.00 \pm 1.09$ & $25.44 \pm 0.51$ \\
\hline Fixed Sequence & $24.23 \pm 0.53$ & $24.88 \pm 0.81$ & $24.56 \pm 1.35$ \\
\hline Smart Explorer & $14.98 \pm 8.73$ & $14.90 \pm 11.35$ & $8.14 \pm 4.91$ \\
\hline Average & 36.27 & 36.14 & 27.88 \\
\hline
\end{tabular}

Table 20: Run Similarity F1-measure of Gaussian Dependent k $=10$ with constant decay

From the data, we see that most of the different means and standard deviations used produce results similar to the previous weights. As the standard deviation or the mean of the weight function increases, the accuracy of the Smart Straight Line behaviour decreases. As we stated in the constant weight section, past information has a higher weighting for a larger mean / standard deviation. This means that past 
information has a negative impact on the similarity of the run.

From the Zig-Zag behaviour, we see a significant increase in accuracy over the TB algorithm when the mean is 1 . At $t=1$, the weight function has the highest value of 1. This means that when the run similarity function is calculating the similarity, the actions and inputs that caused the current case have the highest weighting. From what we can see, the best way to predict the next action is to look at the previous action and input from the current case. This is also true for the NNk2 and BNk2 learning techniques shown in Table 2. When we compare NN to NNk2 and BN to BNk2, we see a large increase in accuracy because NNk2 and BNk2 take into account the previous actions and input when deciding on the next action. 


\begin{tabular}{|c|c|c|c|c|}
\hline & 0 & 1 & 2 & TB k $=10$ \\
\hline \hline Smart Random & $8.74 \pm 0.68$ & $8.77 \pm 0.47$ & $8.68 \pm 0.52$ & $3.02 \pm 0.07$ \\
\hline Smart Straight Line & $8.79 \pm 0.37$ & $8.03 \pm 0.23$ & $8.49 \pm 0.44$ & $3.16 \pm 0.13$ \\
\hline Zig-Zag & $8.32 \pm 0.82$ & $8.10 \pm 0.42$ & $8.18 \pm 0.54$ & $2.96 \pm 0.02$ \\
\hline Fixed Sequence & $4.81 \pm 2.37$ & $5.39 \pm 2.34$ & $8.29 \pm 1.35$ & $2.94 \pm 0.08$ \\
\hline Smart Explorer & $7.63 \pm 0.47$ & $7.65 \pm 0.41$ & $7.69 \pm 0.54$ & $2.90 \pm 0.04$ \\
\hline Average & 7.66 & 7.59 & 8.27 & 3.00 \\
\hline
\end{tabular}

\begin{tabular}{|c|c|c|c|}
\hline & 5 & 10 & TB $\mathrm{k}=10$ \\
\hline \hline Smart Random & $8.58 \pm 0.37$ & $8.63 \pm 0.35$ & $3.02 \pm 0.07$ \\
\hline Smart Straight Line & $8.39 \pm 0.65$ & $8.31 \pm 0.30$ & $3.16 \pm 0.13$ \\
\hline Zig-Zag & $8.43 \pm 0.86$ & $7.99 \pm 0.54$ & $2.96 \pm 0.02$ \\
\hline Fixed Sequence & $8.61 \pm 1.66$ & $10.09 \pm 2.51$ & $2.94 \pm 0.08$ \\
\hline Smart Explorer & $7.54 \pm 0.28$ & $7.59 \pm 0.41$ & $2.90 \pm 0.04$ \\
\hline Average & 8.31 & 8.52 & 3.00 \\
\hline
\end{tabular}

Table 21: Average run time (in milliseconds) of Temporal Backtracking with run similarity using a Gaussian weight function

If we look at the result in Table 21 we can that the run similarity TB has a longer run time than the original TB. 


\subsubsection{Discussion}

In this section, we discussed a similarity function that will compare two runs together and weight cases based on the their temporal offset. We looked at three different weight function: constant weight function, exponential decay weight function, and a Gaussian weight function. When using these weight functions it was found that they gave results that were similar to or worse than the TB algorithm. From these results, we can conclude that when a common weight function is applied, it does not yield a better result than the TB function. Since the same weight function is applied to each candidate run during the comparison, the similarity of each candidate run is adjusted by the same amount and thus differences between the candidate runs remain the same. When we looked at the run time of each of weight functions we saw that the run time of the run similarity is long because it iterates through the whole problem run instead of until the first non-similar case.

\subsection{Edit distance calculation}

The next similarity metric we will look at is edit distance. The edit distance metric [16] will be used as a relative comparison of runs instead of comparisons based on temporal offset. Consider a vacuum robot programmed to move down the hall and stop at the end. In this hall, there is a box that the robot has to avoid. The robot has a sensor at the front of it that will tell the robot if there is an object in front of it and what the object is. If the object is a wall, it will stop; if the object is a box, it will move around it. This produces a trace, $r_{h_{1}}$, as follows: 
$r_{h_{1}}:$ NoObject $\stackrel{\text { Forward }}{\rightarrow}$ NoObject $\stackrel{\text { Forward }}{\rightarrow}$ Box $\stackrel{\text { MoveAround }}{\rightarrow}$ NoObject $\stackrel{\text { Forward }}{\rightarrow}$ Wall $\stackrel{\text { Stop }}{\rightarrow}$

Now let us take the same robot in the same hallway, but in this run, we will remove the box. This produces a the trace, $r_{h_{2}}$, as follows:

$$
r_{h_{2}}: \text { NoObject } \stackrel{\text { Forward }}{\rightarrow} \text { NoObject } \stackrel{\text { Forward }}{\rightarrow} \text { NoObject } \stackrel{\text { Forward }}{\rightarrow} \text { Wall } \stackrel{\text { Stop }}{\rightarrow}
$$

When we compare the second run to the first run we see that the Box input of the first run is removed to produce the second. Alternatively we can say that the Box input was added to the second run to produce the first run. Depending on which run we set as the reference run can affect how we analyze the other run. As a result, we assume that the problem run is error free and the reference run to which all candidate runs are compared to.

As we can see from the traces, the behaviour is reactive, but it highlights some of the things that can occur. To highlight the effect of additions or deletions from noise, consider the example below. We will define the similarity function as follows:

$$
\operatorname{sim}\left(c_{p} c_{c}\right)= \begin{cases}1 & , c_{p}=c_{c} \\ 0 & , \text { otherwise }\end{cases}
$$

- $r_{p}: A \stackrel{a}{\rightarrow} B \stackrel{b}{\rightarrow} C \stackrel{c}{\rightarrow} D \stackrel{?}{\rightarrow}$

- $r_{c_{1}}: A \stackrel{a}{\rightarrow} B \stackrel{b}{\rightarrow} C \stackrel{c}{\rightarrow} B \stackrel{b}{\rightarrow} D \stackrel{e}{\rightarrow}$ 


$$
\text { - } r_{c_{2}}: C \stackrel{e}{\rightarrow} C \stackrel{e}{\rightarrow} B \stackrel{c}{\rightarrow} C \stackrel{c}{\rightarrow} D \stackrel{f}{\rightarrow}
$$

In this example, we see that both candidate runs are longer than the problem run, but there are differences in how similarity equations analyze the different candidate runs. For this example, we will compare the TB algorithm with an edit distance similarity metric described by Algorithm 2.

Input : current run (run), candidate runs (pastRuns)

Output: edit distance (editDist)

1 Function editDistance(run, pastRuns, time) : editDist

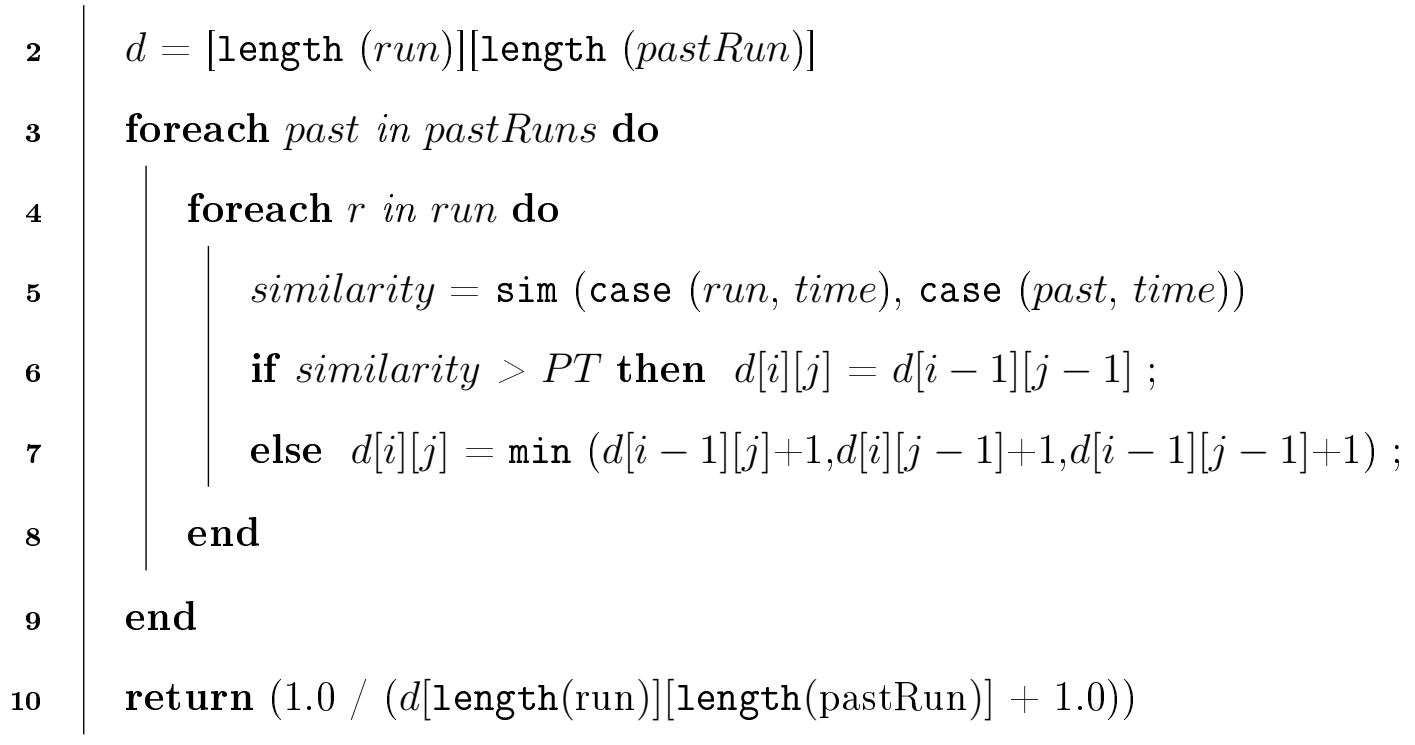

11 end

Algorithm 2: Edit distance algorithm

The algorithm compares each case of the problem run with each case of the candidate run. From this comparison, it calculates the minimum amount of changes from the problem run to the candidate run. The algorithm stores all the edits in a $n+1$ by $m+1$ matrix, $d$, where $n$ is the length of the problem run and $m$ is the length of the candidate run. The first row and column of matrix $d$ are initialized 
using $d_{0, j}=\sum_{k=0}^{j} 1$ and $d_{i, 0}=\sum_{k=0}^{i} 1$. The distance is transformed into a similarity metric by taking the inverse of the distance. One is added to the distance before taking the inverse for the case when the denominator is zero. The zero in the denominator can only occur when both runs being compared are equal. Taking the inverse of the distance is used to restrict the similarity to $[0,1]$.

Each cell in the distance matrix, $d$, is calculated using the following equation:

$$
d_{i j}= \begin{cases}d_{i-1, j-1} & \operatorname{sim}\left(r_{p}(i), r_{c}(j)\right)>P T \\ \min \begin{cases}d_{i-1, j}+1 & \operatorname{sim}\left(r_{p}(i), r_{c}(j)\right) \leq P T \\ d_{i, j-1}+1 & \\ d_{i-1, j-1}+1 & \end{cases} \end{cases}
$$

Where $r_{p}^{c}(i)$ is the $i$ th case in the past of the problem run and $r_{c}^{c}(j)$ is the $j$ th case in the past of the candidate run. If the similarity of both those cases are over the threshold, the edit distance metric deems those cases equal and does not increase the total number of edits. If the cases are below the threshold, increase the amount of edits. The final number of edits is stored at $d_{n+1, m+1}$. We can see the resulting matrix $d$ for each of the candidate runs. The bold value in the matrix is the final number of edits required. 


\begin{tabular}{|c|c||c|c|c|c|c|}
\hline & & $D \stackrel{e}{\rightarrow}$ & $B \stackrel{b}{\rightarrow}$ & $C \stackrel{c}{\rightarrow}$ & $B \stackrel{b}{\rightarrow}$ & $A \stackrel{a}{\rightarrow}$ \\
\hline & 0 & 1 & 2 & 3 & 4 & 5 \\
\hline \hline$D \stackrel{?}{\rightarrow}$ & 1 & 0 & 1 & 2 & 3 & 4 \\
\hline$C \stackrel{c}{\rightarrow}$ & 2 & 1 & 1 & 1 & 2 & 3 \\
\hline$B \stackrel{b}{\rightarrow}$ & 3 & 2 & 1 & 2 & 1 & 2 \\
\hline$A \stackrel{a}{\rightarrow}$ & 4 & 3 & 2 & 2 & 2 & $\mathbf{2}$ \\
\hline
\end{tabular}

\begin{tabular}{|c|c||c|c|c|c|c|}
\hline & & $D \stackrel{f}{\rightarrow}$ & $C \stackrel{c}{\rightarrow}$ & $B \stackrel{c}{\rightarrow}$ & $C \stackrel{e}{\rightarrow}$ & $C \stackrel{e}{\rightarrow}$ \\
\hline & 0 & 1 & 2 & 3 & 4 & 5 \\
\hline \hline
\end{tabular}

\begin{tabular}{|c|c||c|c|c|c|c|}
\hline \hline$D \stackrel{?}{\rightarrow}$ & 1 & 0 & 1 & 2 & 3 & 4 \\
\hline$C \stackrel{c}{\rightarrow}$ & 2 & 1 & 0 & 1 & 2 & 3 \\
\hline$B \stackrel{b}{\rightarrow}$ & 3 & 2 & 1 & 1 & 2 & 3 \\
\hline$A \stackrel{a}{\rightarrow}$ & 4 & 3 & 2 & 2 & 2 & 3 \\
\hline
\end{tabular}

Table 22: Run comparison of the first example. The bold values are the final minimum amount of edits for each candidate run

\begin{tabular}{|c|c|c|}
\hline Run & TB similarity & Edit distance similarity \\
\hline \hline$r_{p}$ & 3 & 0 \\
\hline$r_{c_{1}}$ & 0 & $1 / 3$ \\
\hline$r_{c_{2}}$ & 2 & $1 / 4$ \\
\hline
\end{tabular}

Table 23: Comparison of temporal backtracking algorithm to temporal backtracking with edit distance similarity 
If we take a look at the similarity values shown in Table 23, we see that candidate run $2, r_{c_{2}}$, has a higher similarity value than $r_{c_{1}}$ when the TB similarity function is used. If we use the edit distance similarity function, we see the opposite result. In run 1, we see that a case has been added when compared to the problem run. In this example, the case that was added has shifted the rest of the cases which caused the TB algorithm to stop comparing the previous case. Although logically the first candidate run is more similar to the problem run, the recent case addition makes it not similar. By using the edit distance algorithm, the problem with the TB algorithm and shifts in candidate runs can be eliminated. This is also true for cases that are subtracted from the run. This is shown in the following example:

- $r_{p}: A \stackrel{a}{\rightarrow} B \stackrel{b}{\rightarrow} C \stackrel{c}{\rightarrow} D \stackrel{?}{\rightarrow}$

- $r_{c_{1}}: A \stackrel{a}{\rightarrow} B \stackrel{b}{\rightarrow} D \stackrel{e}{\rightarrow}$

- $r_{c_{2}}: C \stackrel{c}{\rightarrow} C \stackrel{c}{\rightarrow} D \stackrel{e}{\rightarrow}$

\begin{tabular}{|c|c|c|c|c|}
\hline & & $D \stackrel{e}{\rightarrow}$ & $B \stackrel{b}{\rightarrow}$ & $A \stackrel{a}{\rightarrow}$ \\
\hline & 0 & 1 & 2 & 3 \\
\hline \hline$D \stackrel{?}{\rightarrow}$ & 1 & 0 & 1 & 2 \\
\hline$C \stackrel{c}{\rightarrow}$ & 2 & 1 & 1 & 2 \\
\hline$B \stackrel{b}{\rightarrow}$ & 3 & 2 & 1 & 2 \\
\hline$A \stackrel{a}{\rightarrow}$ & 4 & 3 & 2 & $\mathbf{1}$ \\
\hline
\end{tabular}

\begin{tabular}{|c|c||c|c|c|}
\hline & & $D \stackrel{e}{\rightarrow}$ & $C \stackrel{c}{\rightarrow}$ & $C \stackrel{c}{\rightarrow}$ \\
\hline & 0 & 1 & 2 & 3 \\
\hline \hline$D \stackrel{?}{\rightarrow}$ & 1 & 0 & 1 & 2 \\
\hline$C \stackrel{c}{\rightarrow}$ & 2 & 1 & 0 & 1 \\
\hline$B \stackrel{b}{\rightarrow}$ & 3 & 2 & 1 & 1 \\
\hline$A \stackrel{a}{\rightarrow}$ & 4 & 3 & 2 & 2 \\
\hline
\end{tabular}

Table 24: Run comparison of the second example. The bold values are the final minimum amount of edits for each candidate run 


\begin{tabular}{|c|c|c|}
\hline Run & TB similarity & Edit distance similarity \\
\hline \hline$r_{p}$ & 2 & 1 \\
\hline$r_{c_{1}}$ & 0 & $1 / 2$ \\
\hline$r_{c_{2}}$ & 1 & $1 / 3$ \\
\hline
\end{tabular}

Table 25: Comparison of temporal backtracking algorithm to edit distance

As with the previous example we can see that candidate run 2 has the highest similarity with the TB algorithm, but the lowest similarity with the edit distance similarity.

For the edit distance metric, we limited the number of candidate runs to at most 10 for the experiment. This was to reduce the run time of the experiment. The experiment was run on the same six datasets used in earlier experiments. The experiments were run on a dedicated machine with an Intel i7 process with $12 \mathrm{~GB}$ of RAM. The average time for a simulation of each one of the data sets totalled $\sim 31000$, seconds or 8.6 hours. If we were to not restrict the number of candidate runs, the worse-case scenario would be 6000 candidate runs for each instead of 10 candidate runs. If the simulation time increased proportionally with the number of candidate runs, then for 6000 candidate runs, the time for each data set would be 5160 hours, or 215 days. As a result the runs were limited to 10 . 


\begin{tabular}{|c|c|c|c|c|}
\hline & Accuracy & TB Accuracy & F1-Measure & TB F1 \\
\hline \hline Smart Random & $30.48 \pm 0.52$ & $30.63 \pm 0.35$ & $30.46 \pm 0.52$ & $30.62 \pm 0.36$ \\
\hline Smart Straight Line & $51.91 \pm 0.68$ & $93.28 \pm 0.30$ & $51.90 \pm 0.68$ & $50.66 \pm 1.46$ \\
\hline Zig-Zag & $47.94 \pm 0.76$ & $44.53 \pm 1.76$ & $38.90 \pm 0.93$ & $25.44 \pm 0.51$ \\
\hline Fixed Sequence & $25.23 \pm 0.48$ & $25.44 \pm 1.22$ & $25.20 \pm 0.49$ & $24.56 \pm 1.35$ \\
\hline Smart Explorer & $42.10 \pm 12.11$ & $5.50 \pm 6.60$ & $29.10 \pm 5.81$ & $8.14 \pm 4.91$ \\
\hline Average & 39.53 & 39.88 & 35.11 & 27.88 \\
\hline
\end{tabular}

Table 26: Result for edit distance calculation compared against the temporal backtracking algorithm (TB). Accuracy and F1-measure of the TB algorithm are at $k=10$

\begin{tabular}{|c|c|}
\hline & Run Time (Milliseconds) \\
\hline \hline Smart Random & $1043.66 \pm 44.41$ \\
\hline Smart Straight Line & $1002.54 \pm 93.46$ \\
\hline Zig-Zag & $999.54 \pm 51.60$ \\
\hline Fixed Sequence & $905.38 \pm 129.80$ \\
\hline Smart Explorer & $656.11 \pm 385.91$ \\
\hline Average & 921.45 \\
\hline
\end{tabular}

Table 27: Run time milliseconds of the Temporal Backtracking algorithm with edit distance

The results show that the accuracy for the Smart Random and the Fixed Sequence behaviours are similar to that of the TB algorithm. 
From the results, we can see that there is an increase in the performance of the Zig-Zag behaviour when looking at the F1-measure. One possible explanation for this increase is that the edit distance metric was able to accurately predict more $U P$ and $D O W N$ actions than the TB algorithm. Since there is a bias toward the RIGHT and LEFT action, the increase to accuracy is only by a few percentage points, but the increase to F-measure is noticeable.

From the results, we see that the edit distance metric does better at imitating the Smart Explorer behaviour than the TB algorithm. By going back farther than the temporal backtracking algorithm, the edit distance metric is able to better predict the answer.

If we compare the run times show in Table 27 of edit distance to run similarity and TB we can see that the run time is significantly longer. This significant increase in time is due to the comparison between problem and action run being $O(\mathrm{~nm})$ whereas the run similarity comparison is a $O(n)$.

For the Smart Straight Line behaviour we see that the accuracy of the edit distance metric is significantly lower than that of the TB algorithm. One possible explanation for this is due to the Atomic Case similarity calculation coupled with the high threshold. The threshold that was used was set very high, and as a result for two cases to be similar, both the actions and inputs need to be the same. As a result of the high threshold, small variations in inputs or actions leads to cases being not similar.

Consider a possible run below with the simulation described by Equation 15: 


$$
\operatorname{sim}\left(c_{1}, c_{2}\right)= \begin{cases}1 & , c_{1}=c_{2} \\ 0.75 & , \text { otherwise }\end{cases}
$$

- $r_{p}: A \stackrel{a}{\rightarrow} B \stackrel{a}{\rightarrow} C \stackrel{a}{\rightarrow} D \stackrel{b}{\rightarrow} E \stackrel{?}{\rightarrow}$

- $r_{c_{1}}: C \stackrel{a}{\rightarrow} D \stackrel{a}{\rightarrow} F \stackrel{a}{\rightarrow} G \stackrel{b}{\rightarrow} E \stackrel{c}{\rightarrow}$

- $r_{c_{2}}: M \stackrel{c}{\rightarrow} M \stackrel{c}{\rightarrow} M \stackrel{c}{\rightarrow} M \stackrel{c}{\rightarrow} E \stackrel{b}{\rightarrow}$

If we set the threshold to under 0.75 , the edit distance metric will deem candidate run $1, c_{1}$, to be the closer run because it has less differences from the problem run than candidate run $2, c_{2}$ to the problem run. If we change the threshold to a value above 0.75 , then the cases are not equal, and the edit distance will deem the cases to be different cases. Because the inputs in candidate run 1 are different from the problem case, the edit distance metric will treat the cases as the same as those from candidate run 2.

For the Smart Explorer behaviour, we see that the Edit Distance metric does significantly better than the TB algorithm. From Table 28 we see that the F1 score for the STAND action is the highest of all the actions. From the data, we can say that the edit distance was able to perform well only because it could accurately predict the STAND action of the behaviour. 


\begin{tabular}{|c|c|}
\hline Action & Edit Distance F1 \\
\hline \hline STAND & 0.62 \\
\hline DOWN & 0.16 \\
\hline LEFT & 0.17 \\
\hline UP & 0.29 \\
\hline RIGHT & 0.21 \\
\hline
\end{tabular}

Table 28: Edit distance F1 score for each action

\subsection{Jaccard Distance Calculation}

In this section, we will look at a set-based similarity calculation. This similarity metric should be used when case ordering does not matter. One example of this is the Smart Explorer behaviour using the STAND action. The Smart Explorer will only use the STAND action when it has visited each location. The order in which the behaviour visits each location does not matter [17].

The set-based similarity calculation we will use is the Jaccard coefficient. The Jaccard coefficient can be described by Equation 16:

$$
J(A, B)=\frac{|A \cap B|}{|A \cup B|}
$$

where $A$ and $B$ are sets of cases and we can define the set as $A=\{x \mid \forall x \in$ $\left.r, \operatorname{sim}\left(x_{i}, x_{j}\right)<T, i \neq j\right\}$ which states that for cases $x$ in run $r$, the similarity of case $x_{i}$ and case $x_{j}$ are below a given threshold $T$ and $i$ does not equal $j$. The equation 
$J(A, B)$ is bounded to a range of $[0,1]$, which can be used as the similarity metric comparing two different runs.

For the Jaccard similarity metric we will use a $k=10$. The value of $k$ is limited because of the length of time needed to run the experiments. For a comparable analysis of the Jaccard distance similarity, the similarity metric must be run in the same fashion as the TB equation. This means that the Jaccard metric was run using 7-fold cross-validation for each behaviour and used the same dataset that was used in the TB experiment. For the implementation of this similarity, calculating the intersection of sets $A$ and $B$ was done in a naive way. This resulted in the complexity for calculating the intersection to be $O\left(n^{2}\right)$. This led to a total length of time of about 3 hours using the same dedicated machine that ran the DBN and TB experiments.

If we remove the $k$ restraint and compare the problem run to every run and sub-run in the case base and we end up with a worse-case with about 6000 runs for comparison. If we extrapolate the amount of time needed for $k=10$ for the whole case base, we end up with a run time of about 45000 hours or 75 days. 


\begin{tabular}{|c|c|c|c|c|}
\hline & Accuracy & TB Accuracy & F1 Measure & TB F1 \\
\hline \hline Smart Random & $30.75 \pm 0.70$ & $30.63 \pm 0.35$ & $30.74 \pm 0.71$ & $30.62 \pm 0.36$ \\
\hline Smart Straight Line & $51.80 \pm 1.06$ & $93.28 \pm 0.30$ & $51.78 \pm 1.06$ & $50.66 \pm 1.46$ \\
\hline Zig-Zag & $47.10 \pm 0.75$ & $44.53 \pm 1.76$ & $36.47 \pm 1.56$ & $25.44 \pm 0.51$ \\
\hline Fixed Sequence & $24.83 \pm 0.26$ & $25.44 \pm 1.22$ & $24.75 \pm 0.27$ & $24.56 \pm 1.35$ \\
\hline Smart Explorer & $19.58 \pm 20.70$ & $5.50 \pm 6.60$ & $16.82 \pm 9.61$ & $8.14 \pm 4.91$ \\
\hline Average & 34.81 & 39.88 & 32.11 & 27.88 \\
\hline
\end{tabular}

Table 29: Result for Jaccard distance calculation compared against the temporal backtracking algorithm (TB). Accuracy and F1-measure of the TB algorithm are at $k=10$

\begin{tabular}{|c|c|}
\hline & Simulation Time (Seconds) \\
\hline \hline Smart Random & $33.66 \pm 1.23$ \\
\hline Smart Straight Line & $32.04 \pm 2.63$ \\
\hline Zig-Zag & $33.79 \pm 1.29$ \\
\hline Fixed Sequence & $24.66 \pm 7.35$ \\
\hline Smart Explorer & $23.68 \pm 4.22$ \\
\hline Average & 29.56 \\
\hline
\end{tabular}

Table 30: Run time milliseconds of the Temporal Backtracking algorithm with Jaccard distance

From the results, we see that for most of the behaviours except for Smart Explorer, the Jaccard distance metric performed as well as the edit distance metric. To 


\begin{tabular}{|c|c|c|}
\hline Action & Jaccard F1 & Edit Distance F1 \\
\hline \hline STAND & 0.26 & 0.62 \\
\hline DOWN & 0.17 & 0.16 \\
\hline LEFT & 0.13 & 0.17 \\
\hline UP & 0.15 & 0.29 \\
\hline RIGHT & 0.14 & 0.21 \\
\hline
\end{tabular}

Table 31: Comparison of F1 measure of each of the actions for Jaccard and Edit distance metrics

further analyze the difference between the two difference similarity metrics we will examine the ability to predict different actions. This is shown in Table 31 . We can see from the table that the edit distance was able to perform better than the Jaccard metric for the right and up actions. The difference in the up and right actions could be due to the randomness of the behaviour. What is more interesting is the STAND action.

If we compare the run times show in Table 30 of Jaccard distance to run similarity, edit distance, and TB we can see that the run time is significantly less than the edit distance, but longer then run similarity and the original TB. The Jaccard distance run time is shorter than the edit distance because although at worse case set intersection is $O(n m)$ operation, $n$ and $m$ are less than or equal to the length of the problem and candidate run being compared.

Consider a the run below which shows a possible run from when the agent has finished:

$$
\begin{aligned}
& \text { - } r_{p}: B \stackrel{\text { Right }}{\rightarrow} C \stackrel{\text { Up }}{\rightarrow} A \stackrel{\text { Stand }}{\rightarrow} A \stackrel{?}{\rightarrow} \\
& \text { - } r_{c_{1}}: E \stackrel{\text { Left }}{\rightarrow} D \stackrel{\text { Down }}{\rightarrow} A \stackrel{\text { Stand }}{\rightarrow} A \stackrel{\text { Stand }}{\rightarrow}
\end{aligned}
$$




$$
\text { - } r_{c_{2}}: B \stackrel{\text { Right }}{\rightarrow} F \stackrel{U p}{\rightarrow} C \stackrel{U p}{\rightarrow} A \stackrel{\text { Right }}{\rightarrow}
$$

If we break down both candidate runs into a set of cases then we arrive at $c_{1}=\{E \stackrel{\text { Left }}{\rightarrow}$ $, D \stackrel{\text { Down }}{\rightarrow}, A \stackrel{\text { Stand }}{\rightarrow}\}, p=\{B \stackrel{\text { Right }}{\rightarrow}, C \stackrel{U p}{\rightarrow}, A \stackrel{\text { Stand }}{\rightarrow}\}$ and $c_{2}=\{B \stackrel{\text { Right }}{\rightarrow} C \stackrel{U p}{\rightarrow} F \stackrel{U p}{\rightarrow} A \stackrel{\text { Right }}{\rightarrow}\}$. When we apply the Jaccard metric to the candidate run we see that candidate run 1 has a similarity of $1 / 5$ and candidate run 2 has a similarity of $2 / 5$. Because the Jaccard metric works with sets, repeated cases are folded into one case and thus have equal weighting in a run. This is a possible explanation for why the Straight Line behaviour performs worse with Jaccard than the TB algorithm. For similarity algorithms that use runs, having a repeated case in a run is a possible indication that that case should be repeated. This is true for a run where the agent travels in a straight line or for the Smart Explorer behaviour using the STAND action. Because repeated cases are conflated into one case, inferring cases that are repeating becomes harder.

From this example, we can draw the conclusion that if part of the behaviour involves repeating the same case multiple times under a particular condition, the Jaccard metric is not suited for imitating that behaviour. The better similarity metric for imitating that behaviour is the edit distance metric.

\subsection{Discussion}

In this chapter, we looked at redefining the relationship between runs and cases. Previously, a run was composed of cases and a run was a separate entity from a case. In the new definition, a run is composed of cases, but a run is a case. In addition, we also defined a framework for calculating the similarity of runs. 
Using this framework, we have also looked at three different similarity functions: a run similarity function that calculates the average similarity of the run; a edit distance similarity function that will calculate the least amount of edits (additions, deletions or substitutions) required from one run to another; and lastly a set-based similarity function that will calculate the Jaccard distance between two runs.

In the run similarity function, we used three different weight functions, constant weight, exponential decay and Gaussian weight, and analyzed the effects of these weights on the performance of the algorithm. What we concluded was that using a weight function where the weight value is dependent only on time does not yield a better result.

In the edit distance similarity function, we saw that for Smart Random, Zig-Zag and Fixed Sequence behaviours, the similarity function performed similarly to the TB function. For the Smart Explorer behaviour the similarity function was able to yield a significant result, but the result was due to correctly predicting the STAND action.

In the set-based similarity function we used the Jaccard distance metric. We saw that the performance of the set-based similarity function was similar to the edit distance metric with the exception of the Smart Explorer. We concluded that the edit distance metric should be used over the set-based similarity function.

When we looked at the run time of each of the metrics we found that run similarity had the shortest run time followed by the Jaccard distance metric and followed by the edit distance metric. It was found that the metric complexity had a significant impact on the run time. 
Overall, the similarity functions introduced in this section showed either no improvement or worse performance than the TB algorithm. From this, we can conclude that the TB algorithm should be used over the similarity functions mentioned. 


\section{Chapter 6}

\section{Conclusion}

In this final chapter, we will summarize the contributions of this thesis.

\subsection{Summary of Results / Contributions}

1. Reproduction of past research (Section 4.1): Previous research on LFO using a DBN was noted to have a few problems regarding the quality of the results. These problems included missing standard deviations on mean accuracies reported and only accuracy was used. The research was replicated to produce the accuracies and F-measures missing from the research. These values gave a baseline for comparing DBN with the TB algorithm.

2. Comparison of the DBN framework to TB (Section 4.2): An evaluation of the TB algorithm was performed against the benchmark. From the experiments, it was found that the TB algorithm performed worse than the graphical model solutions in almost all behaviours. We concluded that for the agents in the benchmark, the graphical solution is better. 
3. Analysis of the TB framework (Section 4.3): The algorithm proposed by Floyd [7] has a number of undefined behaviours under certain situations. These situations included consensus not being reached, premature elimination of candidate runs and variable candidate run lengths. By analyzing these situations, possible solutions to these problems were proposed. In addition, we concluded that the TB algorithm was part of a more general family of solutions for non-reactive LFO.

4. Defining a generalized framework for similarity of runs (Section 5.1): A new framework was proposed as a generalized way to handle non-reactive LFO with CBR. A run was redefined to be a case that is composed of cases. In addition, the strategy pattern was introduced to allow each case or run to have its own similarity calculation.

5. Analysis of similarity metrics (Section 5.2, 5.3, 5.4): Three different similarity metrics, run similarity, edit distance and Jaccard distance, were presented as alternatives to the TB algorithm. Overall, from these three alternatives, no improvement was found over the TB algorithm.

\subsection{Future Work}

This work has analyzed the TB algorithm and looked at possible alternatives for the algorithm using a generalized framework. However, there are some open problems and limitations with the work. Some of the limitations and problems are:

- Continuous domain: In the analysis of the DBN and TB algorithms, the domain 
used was a discrete domain. Future work should look at whether a change from discrete to continuous still results in the same conclusion.

- Multiple similarity metrics: While analyzing the new framework, one similarity was used for a run. Because runs can be composed of sub-runs, future work should analyze whether targeted similarity functions would result in a better performance for complex behaviours like Smart Explorer.

- Extending the benchmark: The scenarios used in the benchmark do not cover all situations, and this can explain why very different similarity metrics still reported similar results. In particular, a noisy environment might show the usefulness of the edit distance. Also, too much randomness introduced wide standard deviations, which made it difficult to compare approaches.

- Increasing the training set: The training set used in the benchmark is small. This lead to the STAND action being taken when the behaviour does not take that action. Future work should include increasing the training set and run the EM algorithm on DBN and IOHMM for more iterations. 


\section{List of References}

[1] S. Ontañón, J. L. Montaña, and A. J. Gonzalez, "A dynamic bayesian network framework for learning from observation," in Advances in Artificial Intelligence (C. Bielza, A. Salmern, A. Alonso-Betanzos, J. Hidalgo, L. Mart Ãnnez, A. Troncoso, E. Corchado, and J. Corchado, eds.), vol. 8109 of Lecture Notes in Computer Science, pp. 373-382, Springer Berlin Heidelberg, 2013.

[2] T. Steffens, "Similarity-based opponent modelling using imperfect domain theories," in Proceedings of the 2005 IEEE Symposium on Computational Intelligence and Games (CIG05), Essex University, Colchester, Essex, UK, 4-6 April, 2005, 2005 .

[3] B. D. Argall, S. Chernovab, M. Veloso, and B. Browninga, "A survey of robot learning from demonstration," Robotics and Autonomous Systems, vol. 57, pp. 469-483, 2009.

[4] M. Wooldridge, An Introduction to MultiAgent Systems. John Wiley \& Sons Ltd, second ed., 2009.

[5] B. Argall, B. Browning, and M. Veloso, "Learning robot motion control from demonstration and human advice," in the AAAI Spring Symposium, 2009.

[6] S. Ontañón, J. L. Montaña, and A. J. Gonzalez, "A dynamic-bayesian network framework for modeling and evaluating learning from observation," Expert Systems with Applications, vol. 41, no. 11, pp. 5212 - 5226, 2014.

[7] M. W. Floyd, A General-Purpose Framework for Learning by Observation. PhD thesis, Carleton University, 2013. 
[8] M. Fagan and P. Cunningham, "Case-based plan recognition in computer games," in Case-Based Reasoning Research and Development, pp. 161-170, Springer, 2003.

[9] H. H. Bui, "A general model for online probabilistic plan recognition," in In Proc. of the International Joint Conference on Artificial Intelligence (IJCAI), 2003.

[10] S. Russell and P. Norvig, Artificial Intelligence A Modern Approach. Pearson, third ed., 2010.

[11] T. Dietterich, "Machine learning for sequential data: A review," in Structural, Syntactic, and Statistical Pattern Recognition (T. Caelli, A. Amin, R. Duin, D. de Ridder, and M. Kamel, eds.), vol. 2396 of Lecture Notes in Computer Science, pp. 15-30, Springer Berlin Heidelberg, 2002.

[12] Z. Ghahramani, "Learning dynamic bayesian networks," in Adaptive Processing of Sequences and Data Structures (C. Giles and M. Gori, eds.), vol. 1387 of Lecture Notes in Computer Science, pp. 168-197, Springer Berlin Heidelberg, 1998.

[13] A. Aamodt and E. Plaza, "Case-based reasoning: Foundational issues, methodological variations, and system approaches," AI Communications, vol. 7, no. 1, pp. 39-59, 1994.

[14] S. Tan, "Neighbor-weighted k-nearest neighbor for unbalanced text corpus," $E x$ pert Systems with Applications, vol. 28, no. 4, pp. $667-671,2005$.

[15] E. Gamma, R. Helm, R. Johnson, and J. Vlissides, Design Patterns: Elements of Reusable Object-oriented Software. Boston, MA, USA: Addison-Wesley Longman Publishing Co., Inc., 1995.

[16] R. A. Wagner and M. J. Fischer, "The string-to-string correction problem," J. $A C M$, vol. 21, pp. 168-173, Jan. 1974.

[17] S. Niwattanakul, J. Singthongchai, E. Naenudorn, and S. Wanapu, "Using of jaccard coefficient for keywords similarity," Lecture Notes in Engineering and Computer Science, 2013. 